\title{
ON GOOD UNIVERSALITY AND THE RIEMANN HYPOTHESIS
}

\author{
RADHAKRISHNAN NAIR ${ }^{\ddagger}$, JEAN-LOUIS VERGER-GAUGRY $^{\diamond}$, AND MICHEL WEBER ${ }^{\dagger}$
}

\begin{abstract}
We use subsequence and moving average ergodic theorems applied to Boole's transformation and its variants and their invariant measures on the real line to give new characterisations of the Lindelöf Hypothesis and the Riemann Hypothesis. These ideas are then used to study the value distribution of Dirichlet $L$-functions, and the zeta functions of Dedekind, Hurwitz and Riemann and their derivatives. This builds on earlier work of R. L. Adler and B. Weiss, M. Lifshits and M. Weber, J. Steuding, J. Lee and A. I. Suriajaya using Birkhoff's ergodic theorem and probability theory.
\end{abstract}

Keywords: Ergodic Averages, Boole's Transformation, Dedekind Zeta Function, Dirichlet $L$-function, $L$-Series, Hurwitz Zeta Function, Riemann Zeta Function, The Lindelöf Hypothesis, The Riemann Hypothesis.

2020 Mathematics Subject Classification: 11M06, 28D05, 37A44, 11M26, 11M35, 30D35.

\section{CONTENTS}

1. Introduction 2

2. Applications of Theorem $1.1 \quad 6$

2.1. The Riemann zeta function 6

2.2. Dirichlet $L$-functions $\quad 10$

2.3. The Dedekind zeta function of a number field 11

2.4. The Hurwitz zeta function $\quad 12$

3. Proof of Theorem $1.1 \quad 12$

4. Proof of Theorems 1.2 to $1.5 \quad 14$

5. Comparing dynamical and probabilistic models 21

6. Hartman uniformly distributed and good universal sequences 24

7. Moving averages 26

7.1. Stoltz sequences and Theorem 7.2 26

7.2. Applications and moving average ergodic Theorems 27

8. Sublinearity and the Riemann zeta function 28

Acknowledgements $\quad 29$

References $\quad 29$ 


\section{INTRODUCTION}

Equivalent statements of the Riemann Hypothesis are well-known and can be found e.g. in Titchmarsh [T] or more recently e.g. in Mazur and Stein [MSt]. The Lindelöf Hypothesis asserts that for every $\varepsilon>0$ we have

$$
|\zeta(1 / 2+i t)|=O\left(|t|^{\varepsilon}\right) \quad \text { as }|t| \rightarrow \infty,
$$

where $\zeta(z)$ is the Riemann zeta funtion, and Landau's notation " $O$ " means that there exists a neighbourhood $\mathscr{V}$ of $\infty$ and a constant $c \geq 0$ such that: $|\zeta(1 / 2+i t)| \leq c|t|^{\varepsilon}$ for all $t \in \mathscr{V}$.

Before stating the main Theorems 1.1-1.5, and Theorem 7.2 in the context of moving average ergodic theorems, let us make precise the context in probability theory.

Let $\left(X_{i}\right)_{i \geq 1}$ be a sequence of independent Cauchy random variables, with characteristic function $\phi(t)=e^{|t|}$ and consider the partial sums $S_{n}=X_{1}+\ldots+X_{n}(n=1,2, \ldots)$.

M. Lifshits and M. Weber [LW3] studied the value distribution of the Riemann zeta function $\zeta(s)$ sampled along the random walk $\left(S_{n}\right)_{n \geq 1}$ showing, for $b>2$, that

$$
\lim _{N \rightarrow \infty} \frac{1}{N} \sum_{n=1}^{N} \zeta\left(\frac{1}{2}+i S_{n}\right)=1+o\left(\frac{(\log N)^{b}}{N^{\frac{1}{2}}}\right) .
$$

They also showed that

$$
\left\|\sup _{n \geq 1} \frac{\left|\sum_{q=1}^{n} \zeta\left(\frac{1}{2}+i S_{q}\right)-n\right|}{n^{\frac{1}{2}}(\log n)^{b}}\right\|_{2}<\infty .
$$

Here of course $f(x)=o(g(x))$ means $\lim _{x \rightarrow \infty} \frac{f(x)}{g(x)}=0$. This result was extended to $L$ functions and Hurwitz zeta functions by T. Srichan [Sr]. In [St2] J. Steuding replaced $\left(S_{n}\right)_{n \geq 1}$ in [LW3] by $\left(T^{n} x\right)_{n \geq 1}$ for almost all $x$ on $\mathbb{R}$ with respect the Lebesgue measure, for the Boolean dynamical system by $T x:=x-\frac{1}{x}$. This result has its roots in the observation, due to G. Boole [Bo ], subsequently developed by J.W.L. Glashier [G1] [G2], that if $f$ is integrable on the real numbers, then

$$
\int_{-\infty}^{\infty} f(x) d x=\int_{-\infty}^{\infty} f\left(x-\frac{1}{x}\right) d x .
$$

See $[\mathbf{A d W}]$ for a proof of the ergodicity of this dynamical system. In Theorem 3.1 in [LS], the maps

$$
T_{\alpha, \beta}(x)=\left\{\begin{array}{cl}
\frac{\alpha}{2}\left(\frac{x+\beta}{\alpha}-\frac{\alpha}{x-\beta}\right) & \text { if } x \neq \beta, \\
\beta & \text { if } x=\beta,
\end{array}\right.
$$

for $\alpha>0$ and real $\beta$, are shown to be measure preserving and ergodic with respect to the probability measure

$$
\mu_{\alpha, \beta}(A)=\frac{\alpha}{\pi} \int_{A} \frac{d t}{\alpha^{2}+(t-\beta)^{2}}
$$

for any Lebesgue measureable subset $A$ of the real numbers.

As noted in [LS], if $\lambda$ denotes Lebesgue measure then $\mu_{\alpha, \beta}(A) \leq \frac{1}{\alpha \pi} \lambda(A)$ for all $A \in \mathscr{B}$, where $\mathscr{B}$ denotes the Lebesgue $\sigma$-algebra. Also if $\phi_{\alpha, \beta}(x)=\alpha x+\beta$, with $T=T_{1,0}$ and 
$\mu=\mu_{1,0}$ we have

$$
T_{\alpha, \beta}=\phi_{\alpha, \beta} \circ T \circ \phi_{\alpha, \beta}^{-1}
$$

which implies the $\mu_{\alpha, \beta}$-measure preservation and ergodicity of $T_{\alpha, \beta}$. The referee of this paper has brought to the authors' attention the paper [MSr], which considers a family of rational maps more general than that in $[\mathbf{L S}]$, which we consider in this paper. The suggestion is that our methods in this paper will adapt to the more general framework in [MSr] - at least to a degree. We will not however explore this possibility in this paper, as it would be too much of a digression.

We consider a number of general definitions.

We say $\left(k_{n}\right)_{n \geq 1} \in \mathbb{N}$ is $L^{p}$-good universal if for each dynamical system $(X, \beta, \mu, T)$ and for each $g \in L^{p}(X, \beta, \mu)$ the limit

$$
\ell_{T, g}(x)=\lim _{N \rightarrow \infty} \frac{1}{N} \sum_{n=0}^{N-1} g\left(T^{k_{n}} x\right)
$$

exists $\mu$ almost everywhere.

Also throughout the rest of this paper $\mathscr{B}$ will be the Lebesgue $\sigma$-algebra on $\mathbb{R}$ and when a $\sigma$-algebra on $\mathbb{R}$ is not explicitly mentioned it is in fact $\mathscr{B}$.

We say that a sequence $x_{1}, \ldots, x_{N}, \ldots$ is uniformly distributed modulo one if

$$
\lim _{N \rightarrow \infty} \frac{1}{N} \#\left\{1 \leq n \leq N:\left\{x_{n}\right\} \in I\right\}=|I|
$$

for every interval $I \subseteq[0,1)$. For a real number $y$ we have used $\{y\}$ to denote its fractional part and let $[y]=y-\{y\}$ denote its integer part. We say a sequence of integers is uniformly distributed on $\mathbb{Z}$ if it is uniformly distributed among the residue classes modulo $m$, for each natural number $m>1$. We say a sequence of natural numbers $\left(k_{n}\right)_{n \geq 1}$ is Hartman uniformly distributed (on $\mathbb{Z}$ ) if it is uniformly distributed on $\mathbb{Z}$, and for each irrational number $\alpha$, the sequence $\left(\left\{k_{n} \alpha\right\}\right)_{n \geq 1}$ is uniformly distributed modulo one. This condition coincides with $\left(k_{n}\right)_{n \geq 1}$ being uniformly distributed on the Bohr compactification of $\mathbb{Z}$. Some basics on Hartman uniform distribution can be found in the book of Kuipers and Niederreiter [KN]. Note that if $\left(k_{n}\right)_{n \geq 1}$ is Hartman uniformly distributed on $\mathbb{Z}$, and if for $z$ with $|z|=1$ we set

$$
F(N, z):=\frac{1}{N} \sum_{n=0}^{N-1} z^{k_{n}} \quad(N=1,2, \cdots)
$$

then

$$
\lim _{N \rightarrow \infty} F(N, z)=\left\{\begin{array}{cc}
1 & \text { if } z=1 \\
0 & \text { otherwise }
\end{array}\right.
$$

For a $c \in \mathbb{R}$ we use $\mathbb{H}_{c}$ to denote the half plane $\{z \in \mathbb{C}: \Re(z)>c\}$ and use $\mathbb{L}_{c}$ to denote the line $\{z \in \mathbb{C}: \mathfrak{R}(z)=c\}$. We establish the following theorem.

Theorem 1.1. Let $f$ be a meromorphic function on $\mathbb{H}_{c}$ satisfying the following conditions:

(1) there exists $a K>0$ and $a c^{\prime}>c$ such that for any $t \in \mathbb{R}$, we have

$$
\left|f\left(\left\{\sigma+i t \mid \sigma>c^{\prime}\right\}\right)\right| \leq K
$$


(2) there exists a non-increasing $v:(c, \infty) \rightarrow \mathbb{R}$ such that if $\sigma$ is sufficiently near $c$ then $v(\sigma) \leq 1+c-\sigma$ and that for any $\varepsilon>0$, we have $f(\sigma+i t) \ll_{f, \varepsilon}|t|^{v(\sigma)+\varepsilon}$ as $|t| \rightarrow \infty$;

(3) $f$ has at most one pole of order $m$ in $\mathbb{H}_{c}$ at $s_{0}=\sigma_{0}+i t_{0}$, that is, we can write its Laurent expansion near $s=s_{0}$ as

$$
\frac{a_{-m}}{\left(s-s_{0}\right)^{m}}+\frac{a_{-(m-1)}}{\left(s-s_{0}\right)^{(m-1)}}+\ldots \frac{a_{-1}}{\left(s-s_{0}\right)^{-1}}+a_{0}+\sum_{n=1}^{\infty} a_{n}\left(s-s_{0}\right)^{n}
$$

for $m \geq 0$ where we set $m=0$ if $f$ has no pole in $\mathbb{H}_{c}$.

Then if $\left(k_{n}\right)_{n \geq 1}$ is $L^{p}$-good universal and Hartman uniformly distributed, for any $s \in$ $\mathbb{H}_{c} \backslash \mathbb{L}_{\sigma_{0}}$, we have

$$
\lim _{N \rightarrow \infty} \frac{1}{N} \sum_{n=0}^{N-1} f\left(s+i T_{\alpha, \beta}^{k_{n}}(x)\right)=\frac{\alpha}{\pi} \int_{\mathbb{R}} \frac{f(s+i \tau)}{\alpha^{2}+(\tau-\beta)^{2}} d \tau,
$$

for almost all $x$ in $\mathbb{R}$.

In the case $k_{n}=n(n=1,2, \ldots)$ Theorem 1.1 appears in [LS], where it is shown, using (1), (2) and (3) and contour integration, for

$$
l_{\alpha, \beta}(s):=\frac{\alpha}{\pi} \int_{\mathbb{R}} \frac{f(s+i \tau)}{\alpha^{2}+(\tau-\beta)^{2}} d \tau,
$$

that if $f$ has a pole at $s_{0}=\sigma_{0}+i t_{0}$,

$$
l_{\alpha, \beta}(s)=\left\{\begin{array}{cc}
f(s+\alpha+i \beta)+\widetilde{B_{m}}\left(s_{o}\right), & \text { if } c<\Re(s)<\sigma_{o}, s \neq s_{o}-\alpha-i \beta, \\
\sum_{n=0}^{m} \frac{a_{-n}}{(-2 \alpha)^{n}}, & \text { if } c<\Re(s)<\sigma_{o}, s=s_{o}-\alpha-i \beta, \\
f(s+\alpha+i \beta), & \text { if } \mathfrak{R}(s)>\sigma_{o},
\end{array}\right.
$$

or $l_{\alpha, \beta}(s)=f(s-\alpha+i \beta)$ if $s \in \mathbb{H}_{c}$ and $f$ has no pole. Here $\mathfrak{R}(s)$ denotes the real part of $s$ and

$$
\widetilde{B_{m}}\left(s_{0}\right)=\sum_{n=1}^{m}\left\{\frac{a_{-n}}{i^{n}\left(\beta+i \alpha-i\left(s-s_{0}\right)\right)^{n}}-\frac{a_{-n}}{i^{n}\left(\beta-i \alpha-i\left(s-s_{0}\right)\right)^{n}}\right\} .
$$

Moreover, when $m=1$, we can extend Theorem 1.1 to the line $\mathbb{L}_{\sigma_{0}}$ by setting

$$
l_{\alpha, \beta}\left(\sigma_{0}+i t\right)=f\left(\sigma_{0}+\alpha+i(t+\beta)\right)-\frac{a_{-1} \alpha}{\alpha^{2}+\left(t_{0}-t-\beta\right)^{2}} .
$$

Applications will be given in the next section.

As noted earlier Steuding [St2] showed that if $\zeta$ denotes the Riemann zeta function

$$
\lim _{N \rightarrow \infty} \frac{1}{N} \sum_{n=0}^{N-1} \zeta\left(s+i T^{n} x\right)=\frac{1}{\pi} \int_{\mathbb{R}} \frac{\zeta(s+i \tau)}{1+\tau^{2}} d \tau
$$

exists $\mu$ almost everywhere (for $x$ ). We can measure the stability of these averages another way. Henceforth $C$, possibly with subscripts, will denote a positive constant, though nonnecessarily the same on each occasion. 
Theorem 1.2. Suppose $s=\sigma+i$ with $\frac{1}{2}<\sigma<1$. Let

$$
Y_{N}(\sigma)=Y_{N}(\sigma, x):=\frac{1}{N} \sum_{n=0}^{N-1} \zeta\left(s+i T^{n} x\right) . \quad(N=1,2, \ldots)
$$

Suppose $\left(N_{k}\right)_{k \geq 1}$ is a strictly increasing sequence of positive integers. Then there exists an absolute $C>0$ such that

$$
\sum_{k=1}^{\infty}\left\|\sup _{N_{k} \leq N<N_{k+1}}\left|Y_{N}(s)-Y_{N_{k}}(s)\right|\right\|_{2}^{2} \leq \frac{C}{\pi \sigma}\left|\frac{\zeta(2 \sigma)}{2}+\frac{\zeta(2 \sigma-1)}{2 \sigma-1}\right| .
$$

There are other ergodic theoretic measures of the stability of the Riemann zeta function. In this direction we consider the frequencies associated to the sequences

Evidently

$$
\left(\frac{\zeta\left(s+i T^{n} x\right)}{n}\right)_{n \geq 1}
$$

$$
\lim _{n \rightarrow \infty} \frac{\zeta\left(s+i T^{n} x\right)}{n}=0
$$

for almost all $x$. It is possible to make this more precise as follows. Suppose $0<p<\infty$. For a sequence of real numbers $\underline{x}=\left\{x_{n}: n \geq 1\right\}$ set

$$
\|\underline{x}\|_{p, \infty}:=\left(\sup _{t>0}\left\{t^{p} \#\left\{n:\left|x_{n}\right|>t\right\}\right\}\right)^{\frac{1}{p}} .
$$

Theorem 1.3. (i) Suppose $\frac{1}{2}<\sigma<1$. Then

$$
\lim _{m \rightarrow \infty} \frac{\#\left\{n: \frac{\left|\zeta\left(\sigma+i T^{n} x\right)\right|}{n} \geq \frac{1}{m}\right\}}{m}=\zeta(\sigma+1)-\frac{2}{\sigma(2-\sigma)},
$$

for almost all $x \in \mathbb{R}$. and in $L^{1}(\mu)$ norm.

(ii) Further

$$
\|\|\left\{\frac{\left|\zeta\left(\sigma+i T^{n} x\right)\right|}{n}: n \geq 1\right\}\left\|_{1, \infty}\right\|_{1}<\infty .
$$

Moreover,

Theorem 1.4. Suppose $s=\sigma+i$ with $\frac{1}{2}<\sigma<1$. Suppose $\kappa=\left(k_{n}\right)_{n \geq 1}$ is $L^{p}$ - good universal for $p>2$ and let

$$
R_{N}(s, x, \kappa):=\frac{1}{N} \sum_{n=0}^{N-1} \zeta\left(s+i T^{k_{n}} x\right) . \quad(N=1,2, \ldots) .
$$

Then

(i) there exists a constant $C>0$ such that

$$
\sum_{N=1}^{\infty}\left\|R_{N+1}(s, ., \kappa)-R_{N}(s, ., \kappa)\right\|_{2}^{2} \leq \frac{C}{\pi \sigma}\left|\frac{\zeta(2 \sigma)}{2}+\frac{\zeta(2 \sigma-1)}{2 \sigma-1}\right|,
$$

(ii) there exists $C>0$

$$
\mu_{\alpha, \beta}\left(\left\{x: \sum_{N=1}^{\infty}\left|R_{N+1}(s, x, \kappa)-R_{N}(s, x, \kappa)\right|^{2} \geq \lambda\right\}\right) \leq \frac{C}{\lambda^{2}}\left|\frac{\zeta(2 \sigma)}{2}+\frac{\zeta(2 \sigma-1)}{2 \sigma-1}\right|
$$


We will provide additional information about various special families of sequences in Section 4 and Section 8. On the other hand if we consider the $L^{1}$-norm we have the following theorem in the opposite direction.

Theorem 1.5. Suppose $\kappa=\left(k_{n}\right)_{n>1}$ is Hartman uniformly distributed and $L^{p}$-good universal for fixed $p \geq 1$ and $\sigma \in\left(\frac{1}{2}, 1\right)$. Then

$$
\sum_{N \geq 1}\left|R_{N+1}(\sigma, x, \kappa)-R_{N}(\sigma, x, \kappa)\right|=+\infty
$$

almost everywhere in $x$ with respect to Lebesgue measure.

It would be interesting to get good bounds almost everywhere in $x$ for

$$
\sum_{N=1}^{J}\left|R_{N+1}(\sigma, x, \kappa)-R_{N}(\sigma, x, \kappa)\right|, \quad(J=1,2 \ldots)
$$

even for specific cases of $\kappa$.

The paper is organized as follows: in Section 2 new characterisations of the (extended) Lindelöf Hypothesis are obtained for the Riemann zeta function, and for Dirichlet $L$ functions, Dedekind zeta functions of number fields, Hurwitz zeta functions, as applications of Theorem 1.1. The sequences here are assumed to be Hartman uniformly distributed and $L^{p}$-good universal. Replacing this assumption by being Stoltz leads to similar limit theorems, which are reported in Section 7. The role played by sublinear sequences having controlled growth is investigated in Section 8 for the Riemann zeta function. Section 6 contains some examples of $L^{p}$-good universal sequences and Hartman uniformly distributed sequences. Comparison between the dynamical and probabilistic models of Weber [W], resp. Srichan [Sr], is done in Section 5.

\section{Applications of THEOREM 1.1}

From now, we assume that $\left(k_{n}\right)_{n \geq 1}$ satisfies the hypothesis in Theorem 1.1.

In the sequel, for a function $f$ denote $f^{(0)}=f$ and $f^{(k)}$ the $k$-th derivative of $f$ and set

$$
P_{k}(s):=\frac{(-1)^{k} k !}{i^{k+1}}\left(\frac{1}{(\beta+i \alpha-i(s-1))^{k+1}}-\frac{1}{(\beta-i \alpha-i(s-1))^{k+1}}\right)
$$

for any non-negative integer $k$.

\subsection{The Riemann zeta function.}

Theorem 2.1. Suppose $\left(k_{n}\right)_{n \geq 1}$ is $L^{p}$-good universal for some $p \in[1, \infty]$ and Hartman uniformly distributed. Then for any $k \geq 0$ and $s \in \mathbb{H}_{-\frac{1}{2}} \backslash \mathbb{L}_{1}$ we have

$$
\lim _{N \rightarrow \infty} \frac{1}{N} \sum_{n=0}^{N-1} \zeta^{(k)}\left(s+i T_{\alpha, \beta}^{k_{n}}(x)\right)=\frac{\alpha}{\pi} \int_{\mathbb{R}} \frac{\zeta^{(k)}(s+i \tau)}{\alpha^{2}+(\tau-\beta)^{2}} d \tau
$$

for almost all $x$ in $\mathbb{R}$. 
Denote the right hand side of this limit by $l_{\alpha, \beta}^{(k)}$. Then

$$
l_{\alpha, \beta}^{(k)}=\left\{\begin{array}{cc}
\zeta^{(k)}(s+\alpha+i \beta)+P_{k}(s), & \text { if }-\frac{1}{2}<\mathfrak{R}(s)<1, s \neq 1-\alpha-i \beta, \\
(-1)^{k} \gamma_{k}-\frac{k !}{(2 \alpha)^{k+1}}, & \text { if }-\frac{1}{2}<\mathfrak{R}(s)<1, s=1-\alpha-i \beta, \\
\zeta^{(k)}(s+\alpha+i \beta), & \text { if } \mathfrak{R}(s)>1,
\end{array}\right.
$$

where

$$
\gamma_{k}=\lim _{N \rightarrow \infty}\left(\sum_{n=1}^{N} \frac{\log ^{k} n}{n}-\frac{\log ^{k+1} N}{k+1}\right) .
$$

In the case $k=0$ we can extend the result to the line $\mathbb{L}_{1}$ by setting

$$
l_{\alpha, \beta}^{(0)}=l_{\alpha, \beta}^{(0)}(1+i t)=\zeta^{(0)}(1+\alpha+i(t+\beta))-\frac{\alpha}{\alpha^{2}+\left(t^{2}+\beta^{2}\right)} .
$$

If we set $k_{n}=n(n=1,2, \ldots)$ Theorem 2.1 appears in [LS] and this is in the case $\alpha=$ $1, \beta=0$ and $k=1$ in [St2].

We also mention here that the case $k_{n}=n(n=1,2, \ldots)$ and $T=T_{1,0}$ of Theorems 2.3 and 2.4 to follow, appeared in [St2], of Theorem 2.4 appeared first in [BSY] and of Theorems 2.6 and 2.7 appeared in [LS].

Proof. Note that for $k \geq 0$, we know that $\zeta^{(k)}$ is meromorphic and has an absolutely convergent Dirichlet series for $\Re(s)>1$. Thus the condition (1) of Theorem 1.1 is satisfied for $c^{\prime}>1$. This is because the Laurent expansion of $\zeta$ near the pole at $s=1$ is known [Br] and then that the Laurent expansion of $\zeta^{(k)}$ has the form

$$
\zeta^{(k)}(s)=\frac{(-1)^{k} k !}{(s-1)^{k+1}}+(-1)^{k} \gamma_{k}+\sum_{n=k}^{\infty} \frac{(-1)^{n+1} \gamma_{n}}{(n-k+1) !}(s-1)^{n-k+1} .
$$

Thus if $k \geq 0$ the function $\zeta^{(k)}$ has a pole of order $k+1$ at $s=1$. We can in addition show (Titchmarsh [T], pp. 95-96) that given $\varepsilon>0$ we have

$$
\zeta^{(k)}(\sigma+i t) \ll|t|^{\mu(\sigma)+\varepsilon},
$$

where

$$
\mu(\sigma)=\left\{\begin{array}{cl}
0, & \text { if } \sigma>1, \\
\frac{(1-\sigma)}{2}, & \text { if } 0 \leq \sigma \leq 1, \\
\frac{1}{2}-\sigma, & \text { if } \sigma<0,
\end{array}\right.
$$

if $k \geq 0$. Therefore Theorem 2.1 follows from Theorem 1.1 with $c=-\frac{1}{2}, s_{0}=1$ and $m=k+1$ to $\zeta^{(k)}(s)$ as required.

We now state a formulation of the Lindelöf Hypothesis in terms of $\zeta^{(k)}$ from [LS].

Lemma 2.2. The Lindelöf Hypothesis (1.1) is equivalent, for every $\varepsilon>0$, to

$$
\left|\zeta^{(k)}\left(\frac{1}{2}+i t\right)\right| \ll|t|^{\varepsilon}
$$

as $|t|$ tends to $\infty$, for any $k \geq 0$. 
We now give a new characterization of the Lindelöf Hypothesis for the Riemann zeta function, which generalizes the classical one. The classical case $k_{n}=n(n=0,1,2, \ldots)$ appears in [LS].

Theorem 2.3. Suppose $\left(k_{n}\right)_{n \geq 1}$ is $L^{p}$-good universal for some $p \in[1, \infty]$ and Hartman uniformly distributed. Suppose $k$ is any non-negative integer. Then the statement, for any natural number $l$,

$$
\lim _{N \rightarrow \infty} \frac{1}{N} \sum_{n=0}^{N-1}\left|\zeta^{(k)}\left(\frac{1}{2}+i T_{\alpha, \beta}^{k_{n}}(x)\right)\right|^{2 l}=\frac{\alpha}{\pi} \int_{\mathbb{R}} \frac{\left|\zeta^{(k)}\left(\frac{1}{2}+i \tau\right)\right|^{2 l}}{\alpha^{2}+(\tau-\beta)^{2}} d \tau
$$

for $\mu_{\alpha, \beta}$-almost all $x$ in $\mathbb{R}$, is equivalent to the Lindelöf Hypothesis.

Proof. Via Lemma 2.2 the Lindelöf Hypothesis implies that given $\varepsilon>0$ we have

$$
\left|\zeta^{(k)}\left(\frac{1}{2}+.\right)\right|^{2 m} \in L^{p}\left(\mathbb{R}, \mathscr{B}, \mu_{\alpha, \beta}\right)
$$

for each pair of natural numbers $k, m$. Here . represents the variable $t \in \mathbb{R}$. Theorem 1.1 and the ergodicity of $T_{\alpha, \beta}$ implies that

$$
\lim _{N \rightarrow \infty} \frac{1}{N} \sum_{n=0}^{N-1}\left|\zeta^{(k)}\left(\frac{1}{2}+i T_{\alpha, \beta}^{k_{n}}(x)\right)\right|^{2 l}=\frac{\alpha}{\pi} \int_{\mathbb{R}} \frac{\left|\zeta^{(k)}\left(\frac{1}{2}+i \tau\right)\right|^{2 l}}{\alpha^{2}+(\tau-\beta)^{2}} d \tau .
$$

We now prove the converse. First

$$
\zeta^{(k)}(s)=(-1)^{k-1} \int_{1}^{\infty} \frac{[x]-x+\frac{1}{2}}{x^{s+1}}(\log x)^{k-1}(-s \log x+k) d x+\frac{(-1)^{k} k !}{(s-1)^{k+1}} .
$$

Thus there exists $C_{k}>0$, dependent only on $k, \alpha$ and $\beta$, such that for $|t| \geq 1$ we have

$$
\left|\zeta^{(k)}\left(\frac{1}{2}+i t\right)\right|<C_{k}|t|
$$

Also evidently there exists $c_{\alpha, \beta}>0$ such that if $|\tau| \geq 1$

$$
\frac{1}{\alpha^{2}+(\tau-\beta)^{2}} \geq c_{\alpha, \beta} \frac{1}{1+\tau^{2}} .
$$

Assuming that the Lindelöf Hypothesis is false, implies there exists $\eta>0$ and $\tau_{m} \rightarrow \infty$ and $C_{\alpha, \beta}^{1}$ such that

$$
\left|\zeta^{(m)}\left(\frac{1}{2}+i t\right)\right|>C_{\alpha, \beta}^{1} \tau_{m}^{\eta}
$$

Now, $\left|\zeta^{(k)}\left(\frac{1}{2}+i t\right)\right|<C_{k}|t|$ for any $|t| \geq 1$ with $C_{k}>0$, and

$$
\left|\zeta^{(k)}\left(\frac{1}{2}+i \tau\right)-\zeta\left(\frac{1}{2}+i \tau_{m}\right)\right|=\left|\int_{\tau_{m}}^{\tau}\right| \zeta^{(k+1)}\left(\frac{1}{2}+i t\right) d t\left|<C_{\alpha, \beta}^{2}\right| \tau-\tau_{m} \mid \tau .
$$

So $\left|\zeta\left(\frac{1}{2}+i \tau\right)\right| \geq \frac{1}{2} C_{\alpha, \beta}^{1} \tau_{m}^{\eta}$ for $\tau$ with $\left|\tau-\tau_{m}\right| \leq \tau_{m}^{-1}$ with $m$ large enough. Let $L:=\frac{2}{3} \tau_{m}$; then the interval $I:=\left(\tau_{m}-\tau_{m}^{-1}, \tau_{m}+\tau_{m}^{-1}\right)$ contains the interval $(L, 2 L)$ for large $m$. Hence

$$
\int_{L}^{2 L}\left|\zeta^{(k)}\left(\frac{1}{2}+i \tau\right)\right|^{2 l}\left(\frac{1}{2} \frac{d \tau}{1+\tau^{2}}\right) \geq\left(\frac{C_{1}}{2}\right)^{2 l} \int_{I} \tau_{m}^{2 l \eta-2} d \tau=2 .\left(\frac{C_{1}}{2}\right)^{2 l} \cdot \tau_{m}^{2 l \eta-3}
$$


which is $\gg T^{2 l \eta-3}$, and this is impossible as $l \rightarrow \infty$. So our theorem is proved.

We now specialize to the case $T=T_{1,0}$ and give a condition in terms of ergodic averages equivalent to the Riemann Hypothesis. We denote by $\rho=\beta+i \gamma$, a representative complex zeros of the Riemann zeta function. See Titschmarsh [T], p. 30 for instance for details.

Theorem 2.4. Suppose $\left(k_{n}\right)_{n \geq 1}$ is both Hartman uniformly distributed and $L^{p}$-good universal for some $p>1$. Then for almost all $x$ in $\mathbb{R}$ with respect to Lebesgue measure we have

$$
\lim _{N \rightarrow \infty} \frac{1}{N} \sum_{n=1}^{N} \log \left|\zeta\left(\frac{1}{2}+\frac{1}{2} i T^{k_{n}} x\right)\right|=\sum_{\rho: \Re(\rho)>\frac{1}{2}} \log \left|\frac{\rho}{1-\rho}\right| .
$$

Evidently, the Riemann Hypothesis follows if either side is zero.

To prove Theorem 2.4, we need the following lemma due to M. Balazard, E. Saias and M. Yor [BSY].

Lemma 2.5. We have

$$
\frac{1}{2 \pi} \int_{\Re(s)=\frac{1}{2}} \frac{\log |\zeta(s)|}{|s|^{2}}|d s|=\sum_{\rho: \Re(\rho)>\frac{1}{2}} \log \left|\frac{\rho}{1-\rho}\right| .
$$

Proof. We wish to use Theorem 1.1 to deduce Theorem 2.4 using Lemma 2.5. To show Theorem 1.1 is relevant we need to show that

$$
\log \left|\zeta\left(\frac{1}{2}+i .\right)\right| \in L^{p}\left(\mathbb{R}, \mu_{1,0}\right)
$$

(where . denotes $t \in \mathbb{R}$ ), i.e. that

$$
\int_{\mathfrak{R}(s)=\frac{1}{2}}\left(\frac{|\log | \zeta(s))\left.\right|^{p} \mid}{|s|^{2}}\right)|d s|<\infty .
$$

We mentioned earlier that there exists $C>0$ such that if $|t|>1$,

$$
\left|\zeta\left(\frac{1}{2}+i t\right)\right| \leq C|t|
$$

Also notice that $\frac{(\log |\zeta(s)|)^{p}}{|s|^{2}}$ is continuous on an interval on $\Re(s)=\frac{1}{2}$ centred on $s=\frac{1}{2}$. Away from that interval on $\Re(s)=\frac{1}{2}$ we use the observation that $\left|\zeta\left(\frac{1}{2}+i t\right)\right| \leq C|t|$. Hence (for $\left.s=\frac{1}{2}+i t\right)$, given $\delta>0$ we have

$$
\frac{(\log |\zeta(s)|)^{p}}{|s|^{2}} \ll \frac{1}{|s|^{2-\delta}} .
$$

This means

$$
\log \left|\zeta\left(\frac{1}{2}+i .\right)\right| \in L^{p}\left(\mathbb{R}, \mu_{1,0}\right)
$$


as required. Using the fact that $T$ preserves the measure $\mu_{1,0}$ and is ergodic with respect to this measure, we have

$$
\lim _{N \rightarrow \infty} \frac{1}{N} \sum_{n=1}^{N} \log \left|\zeta\left(\frac{1}{2}+\frac{1}{2} i T^{k_{n}} x\right)\right|=\frac{1}{2 \pi} \int_{\Re(s)>\frac{1}{2}} \frac{\log |\zeta(s)|}{|s|^{2}}|d s| .
$$

$\mu_{1,0}$ almost everywhere. Theorem 2.4 now follows from Lemma 2.5.

\subsection{Dirichlet $L$-functions.}

Theorem 2.6. Let $L(s, \chi)$ denote the L-functions associated to the character $\chi$. Suppose $\left(k_{n}\right)_{n \geq 1}$ is Hartman uniformly distributed and $L^{p}$-good universal for some $p>1$. Then, for $k \geq 1$,

(i) if $\chi$ is non-principal, for $s \in \mathbb{H}_{-\frac{1}{2}}$ we have

$$
\begin{aligned}
\lim _{N \rightarrow \infty} \frac{1}{N} \sum_{n=0}^{N-1} L^{(k)}(s+ & \left.i T_{\alpha, \beta}^{k_{n}}(x), \chi\right)=\frac{\alpha}{\pi} \int_{\mathbb{R}} \frac{L^{(k)}(s+i \tau, \chi)}{\alpha^{2}+(\tau-\beta)^{2}} d \tau \\
& =L^{(k)}(s+\alpha+i \beta, \chi) \quad \text { for almost all } x \text { in } \mathbb{R},
\end{aligned}
$$

(ii) if $\chi\left(=\chi_{0}\right)$ is principal, for $s \in \mathbb{H}_{-\frac{1}{2}} \backslash \mathbb{L}_{1}$ we have

$$
\lim _{N \rightarrow \infty} \frac{1}{N} \sum_{n=0}^{N-1} L^{(k)}\left(s+i T_{\alpha, \beta}^{k_{n}}(x), \chi\right)=\frac{\alpha}{\pi} \int_{\mathbb{R}} \frac{L^{(k)}(s+i \tau, \chi)}{\alpha^{2}+(\tau-\beta)^{2}} d \tau,
$$

for almost all $x$ in $\mathbb{R}$.

Denote this limit, i.e. the right hand side of (2.10), by $l_{\alpha, \beta}^{(k)}\left(s, \chi_{0}\right)$. Then

$$
l_{\alpha, \beta}^{(k)}\left(s, \chi_{0}\right)=\left\{\begin{array}{cc}
L^{(k)}\left(s+\alpha+i \beta, \chi_{0}\right)+\gamma_{-1}\left(\chi_{0}\right) P_{k}(s), & \text { if }-\frac{1}{2}<\Re(s)<1, s \neq 1-\alpha-i \beta, \\
\gamma_{k}\left(\chi_{0}\right)-\frac{k ! \gamma_{-1}\left(\chi_{0}\right)}{(2 \alpha)^{k+1}}, & \text { if }-\frac{1}{2}<\Re(s)<1, s=1-\alpha-i \beta, \\
L^{(k)}\left(s+\alpha+i \beta, \chi_{0}\right), & \text { if } \mathfrak{R}(s)>1,
\end{array}\right.
$$

where $\gamma_{-1}\left(\chi_{0}\right), \gamma_{k}\left(\gamma_{0}\right)$, are constants that depend on $\chi_{0}$. These are the coefficients of the Laurent expansion of $L^{(k)}\left(s, \chi_{0}\right)$ near $s=1$. If $k=0$ we can extend the result to the line $\mathbb{L}_{1}$ by defining

$$
l_{\alpha, \beta}^{(0)}=l_{\alpha, \beta}^{(0)}\left(1+i t, \chi_{0}\right)=L\left(1+\alpha+i(t+\beta), \chi_{0}\right)-\frac{\alpha \gamma_{-1}\left(\chi_{0}\right)}{\alpha^{2}+\left(t^{2}+\beta^{2}\right)} .
$$

Proof. We know that $L^{(k)}(s, \chi)$ has a Dirichlet series expansion for $\Re(s)>1$, for each non-negative integer $k$. From this we can show that

$$
\left|L^{(k)}(s, \chi)\right| \ll_{k}, \varepsilon|t|^{\mu(\sigma)+\varepsilon}
$$

where

$$
\mu(\sigma)=\left\{\begin{array}{cc}
0, & \text { if } \sigma>1, \\
\frac{(1-\sigma)}{2}, & \text { if } 0 \leq \sigma \leq 1, \\
\frac{1}{2}-\sigma, & \text { if } \sigma<0 .
\end{array}\right.
$$

If $\chi$ is non-principal then $L^{(k)}(s, \chi)$ is entire for all $k \geq 0$, so $L^{(k)}(s, \chi)$ satisfies Theorem 1.1 for all $s \in \mathbb{H}_{-\frac{1}{2}}$. If $\chi=\chi_{0}$ is principal, $L^{(k)}\left(s, \chi_{0}\right)(k \geq 1)$ has a pole of order $k+1$ at $s=1$. 
We can therefore apply Theorem 1.1 with $c=-\frac{1}{2}, s_{0}=1$ and $m=k+1$, to $L^{(k)}\left(s, \chi_{0}\right)$ with Laurent coefficients coming from [IK].

2.3. The Dedekind zeta function of a number field. We now consider the Dedekind $\zeta_{\mathbb{K}}(s)$ function of a number field $\mathbb{K}$ of degree $d_{\mathbb{K}}$ over the rationals $\mathbb{Q}$ which is defined by as follows. For $s \in \mathbb{C}$ such that $\mathfrak{R}(s)>1$, let

$$
\zeta_{K}(s)=\sum_{I \subset \mathscr{O}_{\mathbb{K}}} \frac{1}{\left(N_{\mathbb{K} / \mathbb{Q}}(I)\right)^{s}} .
$$

Here $I$ runs over the ideals contained in the ring of integers of $K$ denoted $\mathscr{O}_{K}$ and $N_{K / \mathbb{Q}}(I)$ denotes the absolute norm of $I$ in $K$, which is the cardinality of the quotient $\mathscr{O}_{K} / I$. In the case $\mathbb{K}=\mathbb{Q}$, the function $\zeta_{\mathbb{K}}(s)$ reduces to the Riemann zeta function $\zeta(s)$. The complex function $\zeta_{\mathbb{K}}(s)$ can be extended meromorphically to the entire complex plane with a simple pole at $s=1$. See [Coh] p. 216, for more details.

Theorem 2.7. Suppose $\left(k_{n}\right)_{n \geq 1}$ is $L^{p}$-good universal for some $p \in[1, \infty]$ and Hartman

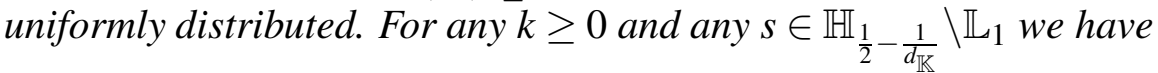

$$
\lim _{N \rightarrow \infty} \frac{1}{N} \sum_{n=0}^{N-1} \zeta_{\mathbb{K}}^{(k)}\left(s+i T_{\alpha, \beta}^{k_{n}}(x)\right)=\frac{\alpha}{\pi} \int_{\mathbb{R}} \frac{\zeta_{\mathbb{K}}^{(k)}(s+i \tau)}{\alpha^{2}+(\tau-\beta)^{2}} d \tau
$$

for almost all $x$ in $\mathbb{R}$.

Denote the right hand side of this limit by $l_{\mathbb{K}_{\alpha, \beta}}^{(k)}$. We have

$$
l_{\mathbb{K}_{\alpha, \beta}}^{(k)}(s)=\left\{\begin{array}{cc}
\zeta_{\mathbb{K}}^{(k)}(s+\alpha+i \beta)+\gamma_{-1}(\mathbb{K}) P_{k}(s), & \text { if } \frac{1}{2}-\frac{1}{d_{\mathbb{K}}}<\mathfrak{R}(s)<1, s \neq 1-\alpha-i \beta, \\
k ! \gamma_{k}(\mathbb{K})-\frac{k ! \gamma_{-1}(\mathbb{K})}{(2 \alpha)^{k+1}}, & \text { if } \frac{1}{2}-\frac{1}{d_{\mathbb{K}}}<\mathfrak{R}(s)<1, s=1-\alpha-i \beta, \\
\zeta^{(k)}(s+\alpha+i \beta), & \text { if } \mathfrak{R}(s)>1 .
\end{array}\right.
$$

Here $\gamma_{-1}(\mathbb{K})$ and $\gamma_{k}(\mathbb{K})$ are constants dependent only on $\mathbb{K}$, which are coefficients of the Laurent expansion of $\zeta_{\mathbb{K}}^{(k)}$ near $s=1$. Also in the case $k=0$ we can extend the result to the line $\mathbb{L}_{1}$ by setting

$$
l_{\mathbb{K}_{\alpha, \beta}}^{(0)}(1+i t)=\zeta^{(0)}(1+\alpha+i(t+\beta))-\frac{\alpha \gamma_{-1}(\mathbb{K})}{\alpha^{2}+\left(t^{2}+\beta^{2}\right)} .
$$

Proof. We refer to [St1] for a bound for $L$ on the half line and to [HIKW] for the coefficients of the Laurent expansion $\zeta_{\mathbb{K}}(s)$ near the pole $s=1$. We then proceed as earlier with Theorem 2.1, $c=\frac{1}{2}-\frac{1}{d_{\mathbb{K}}}, s_{0}=1$ and $m=k+1$.

Remark 2.8. Results analogous to the ergodic characterisation of the Lindelöf Hypothesis just given, can be proved for $L$-functions associated to primitive characters and Dedekind $\zeta$ function. In both cases, the statements of ergodic characterisation are of the type: there exist constants $k, \varepsilon>0$ such that

$$
f\left(\frac{1}{2}+i t\right) \ll_{f, k, \varepsilon}|t|^{\varepsilon},
$$

as $|t| \rightarrow \infty$, for suitable $L$-functions $f$. This inequality when $f$ is an $L$-function arising from Dirichlet series is called the Generalized Lindelöf Hypothesis. 
2.4. The Hurwitz zeta function. Recall that the Hurwitz zeta function is defined for $a>0$ and $s \in \mathbb{C}$ with $\mathfrak{R}(s)>1$ by

$$
\zeta(s, a)=\sum_{n=1}^{\infty} \frac{1}{(n+a)^{s}} .
$$

It is continued meromorphically to the whole of $\mathbb{C}$ with a single pole at $s=1$.

Theorem 2.9. Suppose $\left(k_{n}\right)_{n \geq 1}$ is $L^{p}$-good universal for some $p \in[1, \infty]$ and Hartman uniformly distributed. Then for any such that $\Re(s)>-\frac{1}{2}, s \neq 1$, with $0 \leq a<1$ and $k a$ non-negative integer, we have

$$
\lim _{N \rightarrow \infty} \frac{1}{N} \sum_{n=0}^{N-1} \zeta^{(k)}\left(s+i T_{\alpha, \beta}^{k_{n}}(x), a\right)=\frac{\alpha}{\pi} \int_{\mathbb{R}} \frac{\zeta^{(k)}(s+i \tau, a)}{\alpha^{2}+(\tau-\beta)^{2}} d \tau,
$$

for almost all $x$ in $\mathbb{R}$.

Denote the right hand side of this limit by $l_{\alpha, \beta}^{(k)}(s, a)$. Then

$$
l_{\alpha, \beta}^{(k)}(s, a)=\left\{\begin{array}{cc}
\zeta^{(k)}(s+\alpha+i \beta, a)+P_{k}(s), & \text { if }-\frac{1}{2}<\mathfrak{R}(s)<1, s \neq 1-\alpha-i \beta \\
(-1)^{k} \gamma_{k}(a)-\frac{k !}{(2 \alpha)^{k+1}}, & \text { if }-\frac{1}{2}<\mathfrak{R}(s)<1, s=1-\alpha-i \beta, \\
\zeta^{(k)}(s+\alpha+i \beta, a), & \text { if } \mathfrak{R}(s)>1,
\end{array}\right.
$$

where

$$
\gamma_{k}(a)=\lim _{N \rightarrow \infty}\left(\sum_{n=1}^{N} \frac{\log ^{k}(n+a)}{n+a}-\frac{\log ^{k+1}(N+a)}{k+1}\right) .
$$

In the case $k=0$ we can extend the result to the line $\mathbb{L}_{1}$ by setting

$$
l_{\alpha, \beta}^{(0)}(1+i t, a)=\zeta^{(0)}(1+\alpha+i(t+\beta), a)-\frac{\alpha}{\alpha^{2}+\left(t^{2}+\beta^{2}\right)} .
$$

If we set $k_{n}=n(n=1,2, \ldots)$ Theorem 2.1 appears in [LS] and this is the case $\alpha=1, \beta=0$, and $k=1$ in $[\mathbf{S t 2}]$.

Proof. Following the proof of Theorem 2.1 choose $c=-\frac{1}{2}, s_{0}=1$ and $m=k+1$. For the bound on $\left|\zeta^{(k)}(s, a)\right|$ on the half line and the coefficients of the Laurent series of $\zeta^{(k)}(s, a)$ near $s=1$ we refer to $[\mathbf{S t 1}]$.

\section{PROOF OF THEOREM 1.1}

The proof is in the continuation, and a generalization, of a Theorem of [LS] in which conditions (1), (2) and (3) are used. We first recall a special case of a Theorem of S. Sawyer [Sa]:

Suppose for a dynamical system $\left(\mathbb{R}, \mathscr{B}, \mu_{\alpha, \beta}, T_{\alpha, \beta}\right)$ that $g \in L^{p}\left(\mathbb{R}, \mathscr{B}, \mu_{\alpha, \beta}\right)$ and let $\|g\|=$ $\left(\int_{X}|f|^{p} d \mu_{\alpha, \beta}\right)^{\frac{1}{p}}$. Set

$$
M g(x)=\sup _{N \geq 1}\left|\frac{1}{N} \sum_{n=1}^{N} g\left(s+i T_{\alpha, \beta}^{k_{n}}(x)\right)\right| . \quad \quad(N=1,2, \ldots)
$$


If $\left(k_{n}\right)_{n \geq 1}$ is $L^{p}$-good universal for $p>1$, then there exists $C>0$ such that

$$
\|M g\|_{p} \leq C\|g\|_{p}
$$

Because $\left|\frac{1}{N} \sum_{n=1}^{N} g\left(s+i T_{\alpha, \beta}^{k_{n}}(x)\right)\right| \leq M g(x)(N=1,2, \ldots)$ and $M g \in L^{p}$, the dominated convergence theorem implies

$$
h(x)=\lim _{N \rightarrow \infty} \frac{1}{N} \sum_{n=1}^{N} h\left(s+i T_{\alpha, \beta}^{k_{n}}(x)\right)
$$

exists in $L^{p}$-norm. Our next order of business is to show that $h\left(s+i T_{\alpha, \beta}(x)\right)=h(s+i x)$. Let $U_{\alpha, \beta} g(s+i x)=g\left(s+i T_{\alpha, \beta}(x)\right)$. This is a norm preserving operator on $L^{p}$ as $T_{\alpha, \beta}$ is $\mu_{\alpha, \beta}$ measure preserving. Also let $U_{\alpha, \beta}^{-1}$ denote the $L^{2}$ adjoint of $U_{\alpha, \beta}$. Recall that we say any sequence $\left(c_{n}\right)_{n \in \mathbb{Z}}$ is positive definite if given a bi-sequence of complex numbers $\left(z_{n}\right)_{n \in \mathbb{Z}}$, only finitely many of whose terms are non-zero, we have $\sum_{n, m \in \mathbb{Z}} c_{n-m} z_{n} \overline{z_{m}} \geq 0$. Here $\bar{z}$ is the conjugate of the complex number $z$. Let $\langle a, b\rangle=\int_{\mathbb{R}} a \bar{b} d \mu_{\alpha, \beta}$ (i.e. the standard inner product on $\left.L^{2}\right)$. Notice that $\left(\left\langle U_{\alpha, \beta}^{n} g, g\right\rangle\right)_{n \in \mathbb{Z}}$ is positive definite. Recall that the Bochner-Herglotz theorem $[\mathbf{K t}]$ says that there is a measure $\omega_{g}$ on $\mathbb{T}$, such that

$$
\left\langle U_{\alpha, \beta}^{n} g, g\right\rangle=\int_{\mathbb{T}} z^{n} d \omega_{g}(z)
$$

This tells us that

$$
\begin{gathered}
\left\|\frac{1}{N} \sum_{n=1}^{N} g\left(s+i T_{\alpha, \beta}^{k_{n}+1}(x)\right)-\frac{1}{N} \sum_{n=1}^{N} g\left(s+i T_{\alpha, \beta}^{k_{n}}(x)\right)\right\|_{2} \\
=\int_{\mathbb{T}}\left(2-z-z^{-1}\right)\left|\frac{1}{N} \sum_{n=1}^{N} z^{k_{n}}\right|^{2} d \omega_{g}(z)
\end{gathered}
$$

using the parametrization $z=e^{2 \pi i \theta}$ for $\theta \in[0,1)$, this is

$$
=4 \int_{\mathbb{T}} \sin ^{2}\left(\frac{\theta}{2}\right)\left|\frac{1}{N} \sum_{n=1}^{N} z^{k_{n}}\right|^{2} d \omega_{g}(z) .
$$

Using the fact that $\sin \frac{\theta}{2}=0$ if $\theta=0$ and the fact that $\left(k_{n}\right)_{n \geq 1}$ is Hartman uniformly distributed, by (1.9) and (1.10), we see that $g\left(s+i T_{\alpha, \beta}(x)\right)=g(x)$. A standard fact from ergodic theory is that if $T_{\alpha, \beta}$ is ergodic and $g\left(s+i T_{\alpha, \beta}(x)\right)=g(x)$ for measurable $g$ then $g(x)$ is constant, which must be $\int_{\mathbb{R}} g d \mu_{\alpha, \beta}$. This extends to the $L^{p}$-norm for all $p>1$.

All we have to do now is to show that the pointwise limit is the same as the norm limit, i.e. that $\bar{g}(x)=g(x)=\int_{\mathbb{R}} g d \mu_{\alpha, \beta}$. We consider the sequence of natural numbers $\left(N_{t}\right)_{t \geq 1}$ such that

$$
\left|\frac{1}{N_{t}} \sum_{n=1}^{N_{t}} g\left(s+i T_{\alpha, \beta}^{k_{n}}(x)\right)-\int_{\mathbb{R}} g(x) d \mu_{\alpha, \beta}\right|_{p} \leq \frac{1}{t} .
$$

Thus

$$
\sum_{t=1}^{\infty} \int_{X}\left|\frac{1}{N_{t}} \sum_{n=1}^{N_{t}} g\left(s+i T_{\alpha, \beta}^{k_{n}}(x)\right)-\int_{\mathbb{R}} g(x) d \mu_{\alpha, \beta}\right|^{p} d \mu<\infty .
$$


Fatou's lemma gives

$$
\int_{\mathbb{R}}\left(\sum_{t=1}^{\infty}\left|\frac{1}{N_{t}} \sum_{n=1}^{N_{t}} g\left(s+i T_{\alpha, \beta}^{k_{n}}(x)\right)-\int_{\mathbb{R}} g(x) d \mu_{\alpha, \beta}\right|^{p}\right) d \mu<\infty .
$$

This implies

$$
\sum_{t=1}^{\infty}\left|\frac{1}{N_{t}} \sum_{n=1}^{N_{t}} g\left(s+i T_{\alpha, \beta}^{k_{n}}(x)\right)-\int_{\mathbb{R}} g(x) d \mu_{\alpha, \beta}\right|^{p}<\infty .
$$

almost everywhere. This means

$$
\left|\frac{1}{N_{t}} \sum_{n=1}^{N_{t}} g\left(s+i T_{\alpha, \beta}^{k_{n}}(x)\right)-\int_{X} g(x) d \mu_{\alpha, \beta}\right|=o(1) .
$$

$\mu_{\alpha, \beta}$ almost everywhere. As $\left(k_{n}\right)_{n \geq 1}$ is $L^{p}$-good universal we must have

$$
\lim _{N \rightarrow \infty} \frac{1}{N} \sum_{n=0}^{N-1} g\left(s+i T_{\alpha, \beta}^{k_{n}} x\right)=\int_{\mathbb{R}} g(s+i x) d \mu_{\alpha, \beta}
$$

$\mu_{\alpha, \beta}$ almost everywhere as required to prove Theorem 1.1 .

\section{Proof of Theorems 1.2 to 1.5}

We begin by recalling the spectral regularization method of Lifshits and Weber [LW1] [LW2], and some technical preliminaries. Assume $(X, \beta, v)$ is a measure space with finite measure $v$ and that $S$ is a $v$ measure preserving transformation of $(X, \beta, v)$. For $f \in L^{1}(X, \beta, v)$ let

$$
B_{N}(f)=\frac{1}{N} \sum_{k=0}^{N-1} f \circ T^{k} . \quad(N=1,2, \ldots)
$$

We need the following lemma, due to R. Jones, R. Kaufman, J. Rosenblatt and M. Wierdl [JKRW].

Lemma 4.1. Suppose $\left(N_{p}\right)_{p \geq}$ is a strictly increasing sequence of positive integers. Then there exists an absolute constant $C>0$ such that

$$
\sum_{p=1}^{\infty}\left\|\sup _{N_{p} \leq N<N_{p+1}}\left|B_{N}(f)-B_{N_{p}}(f)\right|\right\|_{2}^{2} \leq C\|f\|_{2} .
$$

Write $\log _{+}(u)=\max (1, \log (u))$ for $u \geq 1$. Let $\omega$ denote the spectral measure associated to the element $f$ and define its regularised measure $\hat{\omega}$ via its Radon-Nykodim derivative by

$$
\frac{d \hat{\omega}}{d x}(x)=\int_{-\pi}^{\pi} Q(\theta, x) \omega(d \theta)
$$

where

$$
Q(\theta, x)=\left\{\begin{array}{cc}
|\theta|^{-1} \log _{+}^{2}\left(\left|\frac{\theta}{x}\right|\right) & \text { if }|x|<|\theta| \\
\theta^{2}|x|^{-3} & \text { if }|\theta| \leq|x| \leq \pi
\end{array}\right.
$$

The following is a theorem of Lifshits and Weber [LW1]. 
Lemma 4.2. Suppose $N_{0}$ and $N_{1}$ with $N_{0}<N_{1}$ are positive integers. Then there exists an absolute constant $C>0$ such that

$$
\left\|\sup _{N_{0} \leq N<N_{1}}\left|B_{N}(f)-B_{N_{0}}(f)\right|\right\|_{2}^{2} \leq C \hat{\omega}\left(\left[\frac{1}{N_{1}} \frac{1}{N_{0}}\right)\right) .
$$

Suppose $0<p<\infty$. For a sequence of real numbers $\underline{x}=\left\{x_{n}: n \geq 1\right\}$ the quantity $\|\underline{x}\|_{p, \infty}$ is defined in (1.16). Also if $r<p$ we have

$$
\|\underline{x}\|_{p, \infty} \leq\|\underline{x}\|_{p} \leq\left(\frac{p}{p-r}\right)^{\frac{1}{p}}\|\underline{x}\|_{p, \infty} .
$$

We also consider

$$
N_{\underline{x}}^{*}=\sup _{m \geq 1} \frac{\#\left\{n: \frac{x_{n}}{n}>\frac{1}{m}\right\}}{m} .
$$

In our considerations, $x_{n}=f\left(\tau^{n}(x)\right)(n=1,2, \ldots)$ and $\underline{x}=O_{f}(x)=\left(f\left(\tau^{n}(x)\right)_{n \geq 1}\right.$ which is the value of $f$ along the orbit of a point $x \in X$. Also let $N_{f}(x)=N_{O_{f}(x)}^{*}$. We have the following Lemma due to I. Assani [As] (see also [W] - we refer to Jamison, Orey and Pruitt [JOP] for more on the random variable case, and $[\mathbf{R R}]$ for Birnbaum-Orlicz spaces and the " $L \log _{+} L "$ notation).

Lemma 4.3. (i) For any non-negative $f \in L^{1}(\mu)$ the function $N_{f}(x)$ is weak- $(p, p)$ for all $p \in(1, \infty)$. Further

$$
\lim _{m \rightarrow \infty} \frac{\#\left\{n: \frac{f\left(\tau^{n}(x)\right)}{n}>\frac{1}{m}\right\}}{m}=\int_{X} f d \mu,
$$

$\mu$ almost everywhere and also in $L^{1}(\mu)$-norm.

(ii) For $f \in L \log _{+} L$

$$
\|\|\left\{\frac{f\left(\tau^{n}(x)\right)}{n}\right\}\left\|_{1, \infty}\right\|_{1}<\infty .
$$

Note that, for two positive constants $C_{1}, C_{2}$,

$$
C_{1} N_{f}(x) \leq\left\|\left\{\frac{f\left(\tau^{n}(x)\right)}{n}, n \geq 1\right\}\right\|_{1, \infty} \leq C_{2} N_{f}(x) .
$$

We now turn to the proof of Theorem 1.2.

By Lemma 4.1,

$$
\sum_{k=1}^{\infty}\left\|\sup _{N_{k} \leq N<N_{k+1}}\left|Y_{N}(\sigma)-Y_{N_{k}}(\sigma)\right|\right\|_{2}^{2} \leq \frac{C}{\pi} \int_{\mathbb{R}} \frac{|\zeta(\sigma+i \tau)|^{2}}{1+\tau^{2}} d \tau,
$$

where as before $C$ is universal. We note that, for $\sigma \in\left(\frac{1}{2}, 1\right)$,

$$
\int_{\mathbb{R}} \frac{|\zeta(\sigma+i t)|^{2}}{1+t^{2}} d t=\int_{0}^{\infty} \frac{\{x\}}{x^{2 \sigma+1}} d x=-\frac{1}{\sigma}\left(\frac{\zeta(2 \sigma)}{2}+\frac{\zeta(2 \sigma-1)}{2 \sigma-1}\right) .
$$


Here again $\{x\}=x-[x]$ denotes the fractional part of $x$. The quantity in the bracket on the right hand side is negative. Further,

$$
\frac{1}{2 \pi} \int_{\mathbb{R}} \frac{\left|\zeta\left(\frac{1}{2}+i t\right)\right|^{2}}{\frac{1}{4}+t^{2}} d t=\log (2 \pi)-\gamma
$$

where $\gamma$ is Euler's constant. See Prop. 7, Cor. 8 and (1.26) in Coffey [C]. Thus

$$
\frac{C}{\pi} \int_{\mathbb{R}} \frac{|\zeta(\sigma+i \tau)|^{2}}{1+\tau^{2}} d \tau \leq \frac{C}{\pi \sigma}\left|\frac{\zeta(2 \sigma)}{2}+\frac{\zeta(2 \sigma-1)}{2 \sigma-1}\right| .
$$

completing the proof of Theorem 1.2.

We now turn to the proof of Theorem 1.3. The first assertion is a consequence of Lemma 4.3 (i). The limit is

$$
\frac{1}{\pi} \int_{\mathbb{R}} \frac{|\zeta(\sigma+i t)|^{2}}{1+\tau^{2}} d t
$$

This integral is a special case of integrals calculated in [St1]. As a consequence of Theorem 1.1 in [St2],

$$
\frac{1}{\pi} \int_{\mathbb{R}} \frac{|\zeta(\sigma+i t)|^{2}}{1+\tau^{2}} d t=\zeta(\sigma+1)-\frac{2}{\sigma(2-\sigma)} .
$$

For the second assertion, it follows from Lemma 4.3 (ii). The function $\zeta(s) \in L \log _{+} L$ is integrable, with respect to the Cauchy measure, because of the estimate

$$
\zeta(\sigma+i t) \ll_{\varepsilon} t^{\frac{1-\sigma}{2}+\varepsilon} .
$$

See Titchmarsh, [T] section 5.1, for the details of this. Therefore Theorem 1.3 is proved as required.

To prove Theorem 1.4 we need the following two lemmas.

Suppose $(X, \beta, \mu)$ is a measure space and that $T: X \rightarrow X$ is a measure preserving map. Let $\left(a_{k}\right)_{k=0}^{\infty}$ be a sequence of natural numbers and for any measurable $f$ on $X$ set

$$
C_{N} f(x):=\frac{1}{N} \sum_{k=0}^{N-1} f\left(T^{a_{k}} x\right), \quad(N=1,2, \cdots)
$$

that is the ergodic averages corresponding to the sequence and let

$$
M f(x):=\sup _{N \geq 1}\left|C_{N} f(x)\right|
$$

Further from the data $(X, \beta, \mu, T)$ and $f$ we construct the ergodic $q$-variation function

$$
V_{q} f(x)=\left(\sum_{N \geq 1}\left|C_{N+1} f(x)-C_{N} f(x)\right|^{q}\right)^{\frac{1}{q}} . \quad(q \geq 1)
$$

Our first lemma is Theorem 1 from [NW1].

Lemma 4.4. Suppose for a sequence of natural numbers $\left(a_{k}\right)_{k=0}^{\infty}$ that for some $p>1$ and $\widetilde{C_{p}}>0$ depending only on $p$ and $(X, \beta, \mu, T)$ we have

$$
\|M f\|_{p} \leq \widetilde{C_{p}}\|f\|_{p}
$$


Then there exists another constant $D_{p}>0$ depending only on $p$ and $(X, \beta, \mu, T)$ such that if $q>1$ then

$$
\left\|V_{q} f\right\|_{p} \leq D_{p}\|f\|_{p}
$$

Further suppose there is a constant $\widehat{C}>0$ depending only on $(X, \beta, \mu, T)$ such that we have

$$
\mu(\{x: M f(x)>\lambda\}) \leq \frac{\widehat{C}}{\lambda} \int_{X}|f| d \mu .
$$

Then there is a constant $D>0$ depending only on $(X, \beta, \mu, T)$ such that if $q>1$ then

$$
\mu\left(\left\{x: V_{q} f(x)>\lambda\right\}\right) \leq \frac{D}{\lambda} \int_{X}|f| d \mu .
$$

Proof. Suppose $(X, \mathscr{L}, \eta)$ denote a finite measure space i.e. $\eta(X)<\infty$. Now, let $\left(T_{n}\right)_{n \geq 1}$ denote a sequence of linear transformations of $L^{p}(X, \mathscr{L}, \eta)$ into measurable functions on $X$, such that each $T_{n}$ is continuous in measure, that is, such that if $\left\|f-f_{m}\right\|_{p}$ tends to 0 as $m$ tends to $\infty$, then $\left\|T_{n} f-T_{n} f_{m}\right\|_{p}$ also tends to 0 as $m$ tends to $\infty$. Set $T^{*} f(x)=$ $\sup _{n \geq 1}\left|T_{n} f(x)\right|$ for $f \in L^{p}(X, \mathscr{L}, \eta)$. We will say the family $\left(T_{n}\right)_{n \geq 1}$ commutes with $w$ : $X \rightarrow X$ if $T^{*} f(w(x)) \leq T^{*} g(x)$ where $g(x)=f(w(x))$ on $X$. Now suppose $\mathscr{F}$ is a family of ergodic $\eta$ preserving transformations on $X$, closed under composition. We will call $\left(T_{n}\right)_{n \geq 1}$ distributive if it commutes with all the elements of some family $\mathscr{F}$ on $X$. We have the following result of S. Sawyer [Sa].

Lemma 4.5. Let $\left(T_{n}\right)_{n \geq 1}$ be a distributive sequence of linear operators on $L^{p}(X, \mathscr{L}, \eta)$, where each $T_{n}$ is continuous in measure and maps $L^{p}(X, \mathscr{L}, \eta)$ to measurable functions on $X$. If $p \in[1,2]$ and if $T^{*} f(x)<\infty \eta$ almost everywhere, then there exists a uniform constant $C>0$ such that

$$
\eta\left(\left\{x: T^{*} f(x) \geq \lambda\right\}\right) \leq \frac{C}{\lambda^{p}} \int_{X}|f(x)|^{p} d \eta,
$$

for all $\lambda>0$ and $f \in L^{p}(X, \mathscr{L}, \eta)$.

The conclusion of Lemma 4.5 is that if $T^{*} f(x)<\infty \eta$ almost everywhere, then the operator $T^{*}$ satisfies a weak $(p, p)$. If there is a $C>0$ such that $\left\|T^{*} f\right\|_{p} \leq C\|f\|_{p}$ we say $T^{*}$ satisfies a strong $(p, p)$ inequality. It is easy to check that strong $(p, p)$ inequalities imply the corresponding weak $(p, p)$ inequalities for the operator $T^{*}$. On the other hand it follows from the Marcinkiewicz interpolation theorem [SW] that a weak $(p, p)$ inequality implies a strong $(q, q)$ inequality if $q>p$. We now specialize (4.1) to the situation where $f$ is defined on $X=\mathbb{R}, \mathscr{L}$ is the Lebesgue algebra on $X=\mathbb{R}$ and $\eta=\mu_{\alpha, \beta}$ :

$$
T_{N} f(x):=\frac{1}{N} \sum_{n=1}^{N} f\left(T_{\alpha, \beta}^{k_{n}}(x)\right) \quad(n=1,2, \ldots)
$$

with $f(x)=\zeta(\sigma+i x)$. One checks readily that $\left(T_{N}\right)_{N \geq 1}$ commutes with $\left(T_{\alpha, \beta}^{n}\right)_{n \geq 1}$ and is therefore distributive. In light of Lemma 4.5, we see that Theorem 1.4 follows by recalling 
our estimate

$$
\int_{\mathbb{R}}|\zeta(\sigma+i x)|^{2} d \mu_{\alpha, \beta} \leq \frac{C}{\pi} \int_{\mathbb{R}} \frac{|\zeta(\sigma+i \tau)|^{2}}{1+\tau^{2}} d \tau \leq \frac{C}{\pi \sigma}\left|\frac{\zeta(2 \sigma)}{2}+\frac{\zeta(2 \sigma-1)}{2 \sigma-1}\right|
$$

arising in (4.6) in the Proof of Theorem 1.3, Theorem 1.4 is proved.

It is possible to say more about specific sequences and families of sequences. Suppose $\mathscr{N}=\left(N_{k}\right)_{k \geq 1}$ and $\left(a_{n}\right)_{n \geq 1}$ are sequences of natural numbers. Consider the definition of $C_{N}$ in (4.7). Let

$$
S f(x)=S(\mathscr{N}, f)(x):=\left(\sum_{k \geq 1}\left|C_{N_{k+1}} f(x)-C_{N_{k}} f(x)\right|^{2}\right)^{\frac{1}{2}} .
$$

In the first instance, we are interested in conditions under which there are constants $C>0$ such that

$$
\|S f\|_{2} \leq C\|f\|_{2} \text {. }
$$

We have the following lemma taken from [N2].

Lemma 4.6. Suppose $1<a \leq \frac{N_{k+1}}{N_{k}} \leq b<\infty$ and $a_{k}=\phi(n)$, where $\phi$ is a non-constant polynomial mapping the natural numbers to themselves. Then there is a constant $C>0$ such that (4.13) holds.

The following lemmas are taken from [NW1].

Lemma 4.7. Suppose $1<a \leq \frac{N_{k+1}}{N_{k}} \leq b<\infty$ and $a_{k}=\phi\left(p_{n}\right)$, where $\phi$ is as in Lemma 4.6 and $p_{n}$ is the $n^{\text {th }}$ rational prime. Then there is a constant $C>0$ such that (4.13) holds.

Let $\theta=\left(\theta_{k}\right)_{k \geq 1}$ be a $\mathbb{Z}$ valued sequence of independent identically distributed random variables with basic probability space $(\Omega, \mathscr{B}, P)$. We assume the $\sigma$-algebra $\mathscr{B}$ is $P$ complete and that there exists $\gamma>0$ such that $\mathbb{E}\left(\left(\theta_{1}^{+}\right)^{\gamma}\right)<\infty$. Here we have used $\theta_{1}^{+}$to denote $\max \left(\theta_{1}, 0\right)$. Consider a strictly increasing sequence $\left(q_{k}\right)_{k \geq 1}$ of natural numbers for which $S$ is a bounded map from $L^{2}$ to itself when $a_{k}=q_{k}$ and $N_{k}=\left[\rho^{k}\right]$ for some $\rho>0$, that is such that

$$
\left\|\left(\sum_{k \geq 1}\left|C_{N_{k+1}}(f)-C_{N_{k}}(f)\right|^{2}(x)\right)^{\frac{1}{2}}\right\|_{2} \leq C\|f\|_{2} .
$$

Also assume there exists $\delta \in(0,1)$ such that

$$
q_{k}=o\left(2^{k^{\delta}}\right)
$$

with

$$
\limsup _{k \rightarrow \infty} \frac{\log q_{2 k}}{\log q_{k}}<\infty
$$

and

$$
P\left(q_{1}+\theta_{1} \geq 0\right)=1
$$

Let

$$
C_{N}^{\theta}(f):=\frac{1}{N} \sum_{k=1}^{N} f\left(T^{q_{k}+\theta_{k}} x\right)
$$


Lemma 4.8. If $\left(q_{k}\right)_{k \geq 1}$ satisfies (4.14), (4.15) and (4.16), then there is a set $\Omega_{0}$ contained in $\Omega$ of full $P$ measure such that if $\omega \in \Omega_{0}$ there exists a constant $C>0$ such that

$$
\left\|\left(\sum_{k \geq 1}\left|C_{N_{k+1}}^{\theta}(f)-C_{N_{k}}^{\theta}(f)\right|^{2}(x)\right)^{\frac{1}{2}}\right\|_{2} \leq C\|f\|_{2} .
$$

Consider two strictly increasing sequences of natural numbers $\left(q_{k}\right)_{k \geq 1}$ and $\mathscr{N}=\left(N_{k}\right)_{k \geq 1}$ such that if $a_{k}=q_{k}$ then (4.14) holds. Let $I_{q}=\left[2^{c_{q}}, 2^{c_{q+1}}\right)$ denote the $q^{\text {th }}$ interval of the form $\left[2^{a}, 2^{a+1}\right)$ containing an element of $\mathscr{N}$. Let $\Phi(N)=\left(\log \left[q_{n}+q+2\right]\right)^{\frac{1}{2}}$ if $N \in I_{q}$ and assume

$$
\sum_{N \in \mathscr{N}} \frac{\Phi^{2}(N)}{N}<\infty
$$

Let $\phi=\left(\phi_{k}\right)_{k>1}$ be a sequence of independent, identically distributed random variables defined on a basic probability space $(\Omega, \beta, P)$ with $\phi_{1} \in L^{1}(\Omega, \beta, P)$ such that

$$
\mathbb{E}\left\{\sup _{N \in \mathbb{N} \backslash\{0\}}\left(\frac{\sum_{k=1}^{N}\left(\phi_{k}-\mathbb{E}\left(\phi_{k}\right)\right)^{2}}{N}\right)^{\frac{1}{2}}\right\}<\infty .
$$

Let

$$
w_{N}^{\phi}(f):=\frac{1}{N} \sum_{k=1}^{N} \phi_{k} f\left(T^{q_{k}} x\right) . \quad(N=1,2, \cdots)
$$

Lemma 4.9. Suppose $\left(q_{k}\right)_{k \geq 1}, \mathscr{N}=\left(N_{k}\right)_{k \geq 1}$ and $\phi=\left(\phi_{k}\right)_{k \geq 1}$ satisfy (4.16), (4.18) and (4.19). Then for almost all $\omega$ in $\Omega$

$$
\left\|\left(\sum_{k \geq 1}\left|w_{N_{k+1}}^{\phi_{k}}(f)-w_{N_{k}}^{\phi_{k}}(f)\right|^{2}\right)^{\frac{1}{2}}\right\|_{2} \leq C\|f\|_{2} .
$$

Some remarks about the nature of condition (4.19) are in order. If $N_{k}=\left[k^{\varepsilon}\right](k=$ $1,2, \ldots)$, with $\varepsilon>1$ then (4.19) reduces to

$$
\sum_{k \geq 1} \frac{\log \left(q_{\left[k^{\varepsilon}\right]}+\log k\right)}{k^{\varepsilon}}<\infty,
$$

which is realised if $q_{k}=O\left(2^{k^{\delta}}\right)$ for $\delta>0$. Also if $N_{k}=2^{k}(k=1,2, \ldots)$ then (4.19) reduces to

$$
\sum_{k \geq 1} \frac{\log \left(q_{2^{k}}+\log k\right)}{2^{k}}<\infty
$$

which is realized if $q_{k}=O\left(2^{k^{\gamma}}\right)$. Given the earlier Lemma of this section these two conditions are not difficult to satisfy.

Set $k_{n}=[g(n)](n=1,2, \ldots)$ where $g$ is a differentiable function from $[0, \infty)$ to itself whose derivative increases with its argument. Let $Z_{M}$ denote the cardinality of the set $\left\{n: k_{n} \leq M\right\}$ and suppose for some function $a:[1, \infty) \rightarrow[1, \infty)$ increasing to infinity as its argument does, that we set

$$
b(M):=\sup _{\{\alpha\} \in\left[\frac{1}{a(M)}, \frac{1}{2}\right)}\left|\sum_{n: k_{n} \leq M} e^{2 \pi i k_{n} \alpha}\right| .
$$


Suppose also for some decreasing function $c:(1, \infty) \rightarrow(0, \infty)$ that

$$
\frac{b(M)+Z_{[a(M)]}+\frac{M}{a(M)}}{Z_{M}} \leq C c(M) .
$$

Then if we have

$$
\sum_{s=1}^{\infty} c\left(\rho^{s}\right)<\infty
$$

for every $\rho>1$ we say $\left(k_{n}\right)_{n \geq 1}$ satisfies condition $H$ (see examples in Section 6). Let $b_{k}=g^{-1}(k)-g^{-1}(k-1)$ where $g^{-1}$ here denotes the inverse function of $g$ on its set of definition. Also suppose that there is a constant $C$ such that

$$
\left(g^{-1}\left(\left[\frac{1}{\{\alpha\}}\right]\right)\right)^{2}\left(\sum_{\left[\frac{1}{\{\alpha\}}\right] N_{k} \geq 1}\left(\frac{1}{g^{-1}\left(N_{k}\right)}\right)^{2}\right) \leq C .
$$

Lemma 4.10. Suppose $\left(k_{n}\right)_{n \geq 1}$ satisfies condition $H$, and (4.21) and that $1<a \leq \frac{N_{k+1}}{N_{k}}<$ $b$. Then (4.16) holds for the corresponding square function.

In $[\mathbf{N}]$ it is shown that examples of sequences of integers which satisfy conditions $\mathrm{H}$ include those given by $g(n)=n^{\omega}$, for non-integer $\omega>1, g(n)=e^{(\log n)^{\gamma}}$ for $\gamma \in\left(1, \frac{3}{2}\right)$ and $g(n)=\alpha_{k} n^{k}+\cdots+\alpha_{1} n+\alpha_{0}$, where the real numbers $\alpha_{1}, \cdots, \alpha_{k}$ are not all multiples of the same real number. The reader will readily verify that these examples also satisfy conditions $\mathrm{H}$ and (4.19). An important point of note is that, as shown in $[\mathbf{N}]$, the ergodic averages, for $a_{n}=k_{n}$ and $f$ in $L^{p}$ with $p>1$ converge to a $T$ invariant limit.

We want to show that if $\left(k_{n}\right)_{n \geq 1}$ satisfies conditions $H,(4.19)$ then (4.13) is satisfied with $1<a \leq \frac{N_{k+1}}{N_{k}} \leq b$.

By identifying it with its characteristic function we may view a strictly increasing sequence of natural numbers as a point in the power set of the natural numbers (i.e. $2^{\mathbb{N}}$ ), or as a point in the Cartesian product $\Pi_{n=1}^{\infty} X_{n}$ where for each natural number $n$, we have set $X_{n}=\{0,1\}$. As a consequence we may put a probability measure on the space of strictly increasing sequences of integers, as a product measure $\pi$ by setting $\pi_{n}(\{1\})=\sigma_{n}$ for $\sigma_{n} \in[0,1]$ and $\pi_{n}(\{0\})=1-\sigma_{n}$ and defining $\pi$ to be the Cartesian product measure $\Pi_{n=1}^{\infty} \pi_{n}$. For a strictly increasing sequence of integers $\left(N_{k}\right)_{k \geq 1}$ suppose also that

$$
\sum_{k \geq 1}\left(\frac{\log _{2} N_{k}}{\sum_{n \leq N_{k}} \sigma_{n}}\right)<\infty,
$$

for arbitrary real $\alpha$ that we have some constant $C$ dependent only on $\pi$ such that

$$
\frac{1}{\left(\sum_{n \leq\left[\frac{1}{\{\alpha\}}\right]} \sigma_{n}\right)^{2}} \sum_{N_{k}\left[\frac{1}{\{\alpha\}}\right] \geq 1}\left(\frac{1}{\sum_{n \leq N_{k}} \sigma_{n}}\right)^{2}<C .
$$

Then with regard to the probability measure just defined, we have the following lemmas.

Lemma 4.11. For ergodic averages with regard to almost all strictly increasing sequences $\left(a_{k}\right)_{k \geq 1}$ with respect to $\pi$, if $\left(N_{k}\right)_{k \geq 1}$ and $\left(\sigma_{k}\right)_{k \geq 1}$ satisfy (4.22) and (4.23) above then (4.16) holds. 
Lemma 4.12. Fix a natural number $d>2$ and let $\left(k_{n}\right)_{n \geq 1}$ denote the set

$$
\Lambda=\left\{n \in \mathbb{N}: n=\sum_{j \geq 1} q_{j} d^{j} \text { with } q_{j} \in\{0,1\}\right\}
$$

ordered by size. Then if $N_{k}=d^{k}(k=1,2, \cdots)$ then (4.13) holds.

For $s=\sigma+i t$ set

$$
C_{N}(s, \zeta, x):=\frac{1}{N} \sum_{n=1}^{N} \zeta\left(\sigma+i T^{k_{n}}(x)\right) \quad(N=1,2, \ldots)
$$

and set

$$
S(s, \zeta, x):=\left(\sum_{k \geq 1}\left|C_{N_{k+1}}(s, \zeta, x)-C_{N_{k}}(s, \zeta, x)\right|^{2}\right)^{\frac{1}{2}} .
$$

We have the following theorem.

Theorem 4.13. If $\sigma \in\left(\frac{1}{2}, 1\right)$ we have $C>0$ such that

$$
\|S(s, \zeta, .)\|_{2}^{2} \leq \frac{C}{\pi \sigma}\left|\frac{\zeta(2 \sigma)}{2 \sigma}+\frac{\zeta(2 \sigma-1)}{2 \sigma-1}\right|
$$

for all the families and sequences $\left(k_{n}\right)_{n \geq 1}$ and $\mathscr{N}$ listed in Lemmas 4.6 - 4.12.

The situation is different if we replace $L^{2}$-norms by $L^{1}$-norms.

Lemma 4.14. Suppose $(X, \beta, \mu, T)$ is an ergodic and measure preserving transformation with $\mu$ non-ergodic. Suppose $\left(k_{n}\right)_{n \geq 1}$ is Hartman uniform distributed and $L^{p}$-good universal for fixed $p \geq 1$. Then for any non-constant function $f$ on $(X, \beta, \mu)$ we set

$$
C_{N}(f)=\frac{1}{N} \sum_{n=1}^{N} f\left(T^{k_{n}}(x)\right) \quad(N=1,2, \ldots) .
$$

Then we have

$$
\sum_{N \geq 1}\left|C_{N+1}(f)-C_{N}(f)\right|=+\infty, \quad \mu \text { almost everywhere. }
$$

Applying Lemma 4.14 to $f(x)=\zeta(\sigma+i x)$ for $\sigma \in\left(\frac{1}{2}, 1\right)$ and $x \in \mathbb{R}$ we get Theorem 1.5.

\section{COMPARING DYNAMICAL AND PROBABILISTIC MODELS}

Let $Y=\Pi_{n=1}^{\infty} \Omega$, that is the space of sequences $\left(X_{1}, X_{2}, \cdots\right)$ in $\Omega$. Let $p_{1}$ denote the projection $p_{1}: Y \rightarrow \Omega$ defined by $p_{1}\left(\left(X_{1}, X_{2}, \cdots\right)\right)=X_{1}$. Also let $S: Y \rightarrow Y$ denote the shift map defined on $Y$ by $S\left(\left(X_{1}, X 2, \cdots,\right)\right)=\left(X_{2}, X_{3}, \cdots\right)$. It is routine to check that $S$ preserves the infinite product measure $\mu^{\infty}=\Pi_{n=1}^{\infty} \mu$ on $Y$. That the shift map $T$ is also ergodic with respect to this infinite product measure is consequence of the Kolmogorov zero one law. Now define $f$ on $Y$ by $f\left(\omega_{1}, \omega_{2}, \cdots\right)=X_{1}\left(\omega_{1}\right)$. This means that $X_{n}(\omega)=f\left(S^{n-1} y\right)$ where $y=\left(\omega_{1}, \omega_{2}, \cdots\right)$ and $S$ denotes the above shift map. Also a simple computation shows that $\int_{Y} f(y) d \mu^{\infty}=\mathscr{E}\left(X_{1}\right)$. This means that the strong law of large numbers follows from an application of Birkhoff's pointwise ergodic theorem and the weak law of large numbers from an application of Von Neumann's norm ergodic theorem respectively.

The upshot of this is that, under quite weak hypotheses, the comparison between the random model described in $[\mathbf{W}]$ and the dynamical model in $[\mathbf{S r}]$ is actually a comparison 
between two different dynamical systems. Now suppose that $\left(k_{n}\right)_{n \geq 1}$ is Hartman uniformly distributed and $L^{p}$ good universal for fixed $p \in[1, \infty)$ and $\mu$ is the Cauchy distribution $\mu_{\alpha, \beta}$ then

$$
\lim _{N \rightarrow \infty} \frac{1}{N} \sum_{n=0}^{N-1} f\left(s+i X_{k_{n}}(\omega)\right)=\frac{\alpha}{\pi} \int_{\mathbb{R}} \frac{f(s+i \tau)}{\alpha^{2}+(\tau-\beta)^{2}} d \tau,
$$

for almost all $\omega$ in $\mathbb{R}$. We can specialise this to

$$
\lim _{N \rightarrow \infty} \frac{1}{N} \sum_{n=1}^{N} \log \left|\zeta\left(\frac{1}{2}+\frac{1}{2} i X_{k_{n}}(\omega)\right)\right|=\sum_{\rho: \Re(\rho)>\frac{1}{2}} \log \left|\frac{\rho}{1-\rho}\right| .
$$

Again, the Riemann Hypothesis follows if either side is zero. As above similar observations can be obtained other zeta functions and $L$-functions.

The condition of good universality is an assumption about all dynamical systems. We don't need to assume so much and can deduce our conclusions from the properties of one transformation. The following Theorem offers that link between the two models. See also [JO ] where similar ideas appear.

Theorem 5.1. Consider two ergodic dynamical systems $\left(X_{1}, \beta_{1}, \mu_{1}, T_{1}\right)$ and $\left(X_{2}, \beta_{2}, \mu_{2}, T_{2}\right)$ both on separable measure spaces. Suppose that $\mu_{1}$ and $\mu_{2}$ are non-atomic. Then iffor a particular sequence of integers $\left(k_{n}\right)_{n \geq 1}$ for each $f_{1} \in L^{p}\left(X_{1}, \beta, \mu_{1}\right)$ for all $p>1$ we have

$$
\lim _{N \rightarrow \infty} \frac{1}{N} \sum_{n=1}^{N} f_{1}\left(T_{1}^{k_{n}} x_{1}\right)=\int_{X_{1}} f_{1}\left(x_{1}\right) d \mu_{1},
$$

$\mu_{1}$ almost everywhere, then the same is true with 1 replaced by 2.

The condition of non-atomicity of $\mu_{1}$ and $\mu_{2}$ is not strictly necessary for the proof but it simplifies the proof somewhat and our intended applications are to non-atomic dynamical sytems.

Proof. Let $(X, \beta, \mu, T)$ denote a dynamical system and for sequence of natural numbers $\left(k_{n}\right)_{n \geq 1}$ let

$$
m(f)=\sup _{N \geq 1}\left|\frac{1}{N} \sum_{n=1}^{N} f\left(T^{k_{n}} x\right)\right|
$$

A special case of a theorem of S. Sawyer [Sa], tells us, after the hypothesis of Theorem 5.1 , with $m=m_{1}$, that there exists $C_{p}^{\prime}>0$ such that

$$
\mu\left(\left\{x_{1} \in X_{1}: m_{1}\left(f_{1}\right)\left(x_{1}\right) \geq \lambda\right\}\right) \leq \frac{C_{p}\left\|f_{1}\right\|_{p}}{\lambda} .
$$

Another way to say this is that the operator $m_{1}$ satisfies a weak- $(p, p)$ bound. A stronger assertion is that there exists $C_{p} *>0$ such that

$$
\left\|m_{1}\left(f_{1}\right)\right\|_{p} \leq C_{p}^{*}\left\|f_{1}\right\|_{p} \text {. }
$$

Here we say $m_{1}$ satisfies a strong- $(p, p)$ inequality. For any fixed $p$, a strong- $(p, p)$ inequality implies the corresponding weak- $(p, p)$ inequality. On the other hand as a consequence of the Marcinkiewicz interpolation theorem [SW] weak- $\left(p_{a}, p_{a}\right)$ implies strong- $\left(p_{b}, p_{b}\right)$ if $p_{a}<p_{b}$. This means that the fact that the weak- $(p, p)$ of $m$ for all $p>1$ is equivalent to it being strong- $(p, p)$ for all $p>1$. 
We now show that inequality (5.1) implies

$$
\left\|m_{2}\left(f_{2}\right)\right\|_{p} \leq C_{p}^{*}\left\|f_{2}\right\|_{p}
$$

Suppose that the dynamical systems $\left(X_{1}, \beta_{1}, \mu_{1}, T_{1}\right)$ and $\left(X_{2}, \beta_{2}, \mu_{2}, T_{2}\right)$ are both ergodic. Now the argument used to deduce Theorem 1.1 from (3.1) together with the ergodicy $\left(X_{2}, \beta_{2}, \mu_{2}, T_{2}\right)$ readily implies the conclusion of Theorem 2.1 .

We now prove (5.5). As both the dynamical systems $\left(X_{1}, \beta_{1}, \mu_{1}, T_{1}\right)$ and $\left(X_{2}, \beta_{2}, \mu_{2}, T_{2}\right)$ are ergodic, the Rokhlin-Halmos lemma says that given any integer $N \geq 1$ and $\varepsilon>0$, there exist sets $E_{i} \subset X_{i}(i=1,2)$ with $E_{i}, T_{i}^{-1} E_{i}, \ldots, T_{i}^{-(N-1)} E_{i}$ disjoint and

$$
\mu_{i}\left(E_{i} \cup T_{i}^{-1} E_{i} \ldots \cup T_{i}^{-(N-1)} E_{i}\right)<1-\varepsilon .
$$

Let $\gamma=\frac{\mu_{1}\left(E_{2}\right)}{\mu_{2}\left(E_{2}\right)}$. Also for a set $D$ and a function $f$ let $f_{D}$ denote the restriction of $f$ to $D$.

Let us observe that there always exists a bijection from $E_{1}$ to $E_{2}$ that preserves the measure from $\mu_{1}$ to $\gamma \mu_{2}$. This is always possible by the measure isomorphism theorem between separable spaces. Let us call $\delta$ such a 1-1 measure map from $E_{1}$ to $E_{2}$. It is not canonical.

Now set

$$
F_{i}^{N}=E_{i} \cup T_{i}^{-1} E_{i} \ldots \cup T_{i}^{-(N-1)} E_{i}, \quad(i=1,2)
$$

and extend the definition of $\left.\Delta\right|_{E_{1}}:=\delta$ to $F_{1}$ by setting $\Delta(x)=\left(T_{2}^{k} \delta T_{1}^{-k}\right)(x)$ for $x \in T^{k} E_{1}$. Now set

$$
W f(y):=f\left(\Delta^{-1} y\right) \gamma^{\frac{1}{p}} .
$$

Notice supp $W f(y) \subset T_{2}^{k} E$ if $\operatorname{supp} f(y) \subset T_{1}^{k} E$. Direct computation now gives for supp $f(y) \subset$ $T_{1}^{k} E$

$$
\int_{T^{k} E_{1}}|f|^{p} d \mu_{1}=\int_{T^{k} E_{2}}|W f|^{p} d \mu_{2}
$$

and so

$$
\int_{F_{1}}|f|^{p} d \mu_{1}=\int_{F_{2}}|W f|^{p} d \mu_{2}
$$

Let

$$
C_{i, l}(f)(x)=\frac{1}{l} \sum_{n=1}^{l} f\left(T_{i}^{k_{n}} x\right), \quad(l=1,2, \ldots)
$$

and set

$$
m_{i, N}(f)(x)=\sup _{1 \leq l \leq N}\left|C_{i, l}(f)(x)\right|, \quad(N=1,2, \ldots)
$$

and evidently

$$
m_{i}(f)(x)=\lim _{N \rightarrow \infty} m_{i, N}(f)(x) .
$$

In proving (5.5) by splitting $f$ into its real and imaginary parts and each of those into their positive and non-negative parts we may assume $f \geq 0$. Suppose $x \in T_{1}^{k} E_{1}$ and for the transformation $T_{1}$ we have $m_{N}(f)(x) \geq 0$. Then there exists $l \in[1, N]$ such that

$$
m_{i, l}(f)(x)=C_{i, l}(f)(x) .
$$

Let $y=W x$. Then a direct computation shows that for the transformation $T_{2}$ we have

$$
C_{2, l}(W f)(y)=W\left(m_{1, N}(f)\right)(x) .
$$


From this we deduce that

$$
m_{2, N}(W f)(y) \geq m_{1, l}(f)(x)
$$

Thus

$$
\left\|m_{2, N}(f)\right\|_{p}^{p}=\int_{X_{2}}\left|m_{2, N}(f)(x)\right|^{p} d \mu_{2}
$$

which is

$$
\leq \int_{F_{2}}\left|m_{2, N}(f)(x)\right|^{p} d \mu_{2}+\varepsilon
$$

and we know $W$ fixes measure on $F_{2}$ and hence $L^{p}$ norms so we have

$$
\begin{gathered}
\int_{F_{2}}\left|W\left(m_{2, N}(f)\right)(y)\right|^{p} d \mu_{2}+\varepsilon=\left\|\left(m_{2, N}(W(f))\right)(y)\right\|_{p}^{p} d \mu_{2}+\varepsilon \\
\leq c\|W(f)\|_{p}^{p}+\varepsilon \leq c\|f\|_{p}^{p} d+\varepsilon .
\end{gathered}
$$

Now let $N \rightarrow \infty$ and let $\varepsilon \rightarrow 0$ and the proof of the theorem is complete.

The ergodicity of the random dynamical system is implied by the Kolmogorov $0-1$ law. If we choose $\mu=\mu_{\alpha, \beta}$, we see that Theorem 1.1 is equivalent to (5.1). From this we deduce all the applications of Theorems 5.1 with $T^{k_{n}}$ replaced by $X_{k_{n}}$.

\section{HARTMAN UNIFORMLY DISTRIBUTED AND GOOD UNIVERSAL SEQUENCES}

In this section we give some examples of $L^{p}$-good universal sequences for some $p \geq 1$. The examples 1, 3-6 are Hartman uniformly distributed. Example 2 is not Hartman uniformly distributed in general.

1.- The natural numbers:

The sequence $(n)_{n=1}^{\infty}$ is $L^{1}$-good universal. This is Birkhoff's pointwise ergodic theorem.

Let $\phi$ be any non-constant polynomial mapping the natural numbers to themselves. Note that if $n \in \mathbb{N}$, then $n^{2} \not \equiv 3 \bmod 4$, so in general the sequences $(\phi(n))_{n=1}^{\infty}$ and $\left(\phi\left(p_{n}\right)\right)_{n=1}^{\infty}$ are not Hartman uniformly distributed. We do, however, know that if $\gamma \in \mathbb{R} \backslash \mathbb{Q}$, then $(\phi(n) \gamma)_{n=1}^{\infty}$ and $\left(\phi\left(p_{n}\right) \gamma\right)_{n=1}^{\infty}$ are uniformly distributed modulo 1.

2. Condition $\mathrm{H}$ :

Sequences $\left(k_{n}\right)_{n=1}^{\infty}$ that are both $L^{p}$-good universal and Hartman uniformly distributed can be constructed as follows. Set $k_{n}=[\tau(n)](n=1,2, \ldots c)$, where $\tau:[1, \infty) \rightarrow[1, \infty)$ is a differentiable function whose derivative increases with its argument. Let $\Omega_{m}$ denote the cardinality of the set $\left\{n: a_{n} \leq m\right\}$, and suppose, for some function $\varphi:[1, \infty) \rightarrow[1, \infty)$ increasing to infinity as its argument does, that we set

$$
\rho(m)=\sup _{\{z\} \in\left[\frac{1}{\varphi(m)}, \frac{1}{2}\right)}\left|\sum_{n: k_{n} \leq m} e\left(z k_{n}\right)\right| .
$$

Suppose also, for some decreasing function $\rho:[1, \infty) \rightarrow[1, \infty)$ and some positive constant $\omega>0$, that

$$
\rho(m)+\Omega_{[\varphi(m)]}+\frac{m}{\varphi(m)} \Omega_{m} \leq \omega \rho(m) .
$$


Then if we have

$$
\sum_{n=1}^{\infty} \rho\left(\theta^{n}\right)<\infty
$$

for all $\theta>0$, we say that $\left(a_{n}\right)_{n=1}^{\infty}$ satisfies condition $\mathrm{H}$, see [N].

Sequences satisfying condition $\mathrm{H}$ are known to be both Hartman uniformly distributed and $L^{p}$-good universal. Specific sequences of integers that satisfy condition $\mathrm{H}$ include $a_{n}=[\tau(n)](n=1,2, \ldots c)$ where:

[I.] $\tau(n)=n^{\gamma}$ if $\gamma>1$ and $\gamma \notin \mathbb{N}$.

[II.] $\tau(n)=e^{\left((\log n)^{\gamma}\right)}$ for $\gamma \in\left(1, \frac{3}{2}\right)$.

[III.] $\tau(n)=b_{k} n^{k}+\ldots b+b_{1} n+b_{0}$ for $b_{k}, \ldots c, b_{1}$ not all rational multiplies of the same real number.

[IV.] Hardy fields: By a Hardy field, we mean a closed subfield (under differentiation) of the ring of germs at $+\infty$ of continuous real-valued functions with addition and multiplication taken to be pointwise. Let $\mathscr{H}$ denote the union of all Hardy fields. Conditions for $\left(a_{n}\right)_{n=1}^{\infty}=([\psi(n)])_{n=1}^{\infty}$, where $\psi \in \mathscr{H}$ to satisfy condition $\mathrm{H}$ are given by the hypotheses of Theorems 3.4, 3.5 and 3.8. in [BKQW]. Note the term ergodic is used in this paper in place of the older term Hartman uniformly distributed.

3. A random example:

Suppose that $S=\left(k_{n}\right)_{n=1}^{\infty}$ is a strictly increasing sequence of natural numbers. By identifying $S$ with its characteristic function $\chi_{S}$, we may view it as a point in $\Lambda=\{0,1\}^{\mathbb{N}}$, the set of maps from $\mathbb{N}$ to $\{0,1\}$. We may endow $\Lambda$ with a probability measure by viewing it as a Cartesian product $\Lambda=\prod_{n=1}^{\infty} X_{n}$, where, for each natural number $n$, we have $X_{n}=\{0,1\}$ and specify the probability $v_{n}$ on $X_{n}$ by $v_{n}(\{1\})=$ $\omega_{n}$ with $0 \leq \omega_{n} \leq 1$ and $v_{n}(\{0\})=1-\omega_{n}$ such that $\lim _{n \rightarrow \infty} \omega_{n} n=\infty$. The desired probability measure on $\Lambda$ is the corresponding product measure $v=\prod_{n=1}^{\infty} v_{n}$. The underlying $\sigma$-algebra $\mathscr{A}$ is that generated by the cylinders

$$
\left\{\left(\Delta_{n}\right)_{n=1}^{\infty} \in \Lambda: \Delta_{n_{1}}=\alpha_{n_{1}}, \ldots c, \Delta_{n_{k}}=\alpha_{n_{k}}\right\}
$$

for all possible choices of $n_{1}, \ldots c, n_{k}$ and $\alpha_{n_{1}}, \ldots c, \alpha_{n_{k}}$. Then almost every point $\left(a_{n}\right)_{n=1}^{\infty}$ in $\Lambda$, with respect to the measure $v$, is Hartman uniformly distributed (see Proposition 8.2 (i) in Bourgain [Bou ]). Hartman uniformly distributed sequences are called ergodic sequences in [Bou ].

4. Block sequences:

Suppose that $\left(a_{n}\right)_{n=1}^{\infty}=\bigcup_{n=1}^{\infty}\left[d_{n}, e_{n}\right]$ is ordered by absolute value for disjoint $\left(\left[d_{n}, e_{n}\right]\right)_{n=1}^{\infty}$ with $d_{n-1}=O\left(e_{n}\right)$ as $n$ tends to infinity. Note that this allows the possibility that $\left(a_{n}\right)_{n=1}^{\infty}$ is zero density. This example is an immediate consequence of Tempelman's semigroup ergodic theorem. See page 218 of [BL]. Being a group average ergodic theorem this pointwise limit must be invariant, which ensures that the block sequence must be Hartman uniformly distributed.

5. Random perturbation of good sequences:

Suppose that $\left(a_{n}\right)_{n=1}^{\infty}$ is an $L^{p}$-good universal sequence which is also Hartman uniformly distributed. Let $\theta=\left(\theta_{n}\right)_{n=1}^{\infty}$ be a sequence of $\mathbb{N}$-valued independent, identically distributed random variables with basic probability space $(Y, \mathscr{A}, \mathscr{P})$, and a $\mathscr{P}$-complete $\sigma$-field $\mathscr{A}$. Let $\mathbb{E}$ denote expectation with respect to the basic 
probability space $(Y, \mathscr{A}, \mathscr{P})$. Assume that there exist $0<\alpha<1$ and $\beta>1 / \alpha$ such that

$$
a_{n}=O\left(e^{n^{\alpha}}\right) \mathbb{E} \log _{+}^{\beta}\left|\theta_{1}\right|<\infty .
$$

Then $\left(k_{n}+\theta_{n}(\omega)\right)_{n=1}^{\infty}$ is both $L^{p}$-good universal and Hartman uniformly distributed [NW2].

\section{Moving AVERAGES}

In this paragraph we show that the assumption of a (univariate) sequence which has the property to be $L^{p}$ good universal and Hartman uniformly distributed can be removed from the Theorems 2.1, 2.3, 2.4, 2.6, 2.7, 2.9, and replaced by the assumption of a (bivariate) Stoltz sequence.

7.1. Stoltz sequences and Theorem 7.2. Let $Z$ be a collection of points in $\mathbb{Z} \times \mathbb{N}$ and let

$$
\begin{gathered}
Z^{h}:=\{(n, k):(n, k) \in Z \text { and } k \geq h\}, \\
Z_{\alpha}^{h}:=\left\{(z, s) \in \mathbb{Z}^{2}:|z-y|<\alpha(s-r) \text { for some }(y, r) \in Z^{h}\right\}
\end{gathered}
$$

and

$$
Z_{\alpha}^{h}(\lambda):=\left\{n:(n, \lambda) \in Z_{\alpha}^{h}\right\} \quad(\lambda \in \mathbb{N}) .
$$

Geometrically we can think of $Z_{\alpha}^{1}$ as the lattice points contained in the union of all solid cones with aperture $\alpha$ and vertex contained in $Z^{1}=Z$. We say a sequence of pairs of natural numbers $\left(n_{l}, k_{l}\right)_{l=1}^{\infty}$ is Stoltz if there exists a collection of points $Z$ in $\mathbb{Z} \times \mathbb{N}$, and a function $h=h(t)$ tending to infinity with $t$ such that $\left(n_{l}, k_{l}\right)_{l=t}^{\infty} \in Z^{h(t)}$ and there exist $h_{0}, \alpha_{0}$ and $A>0$ such that for all integers $\lambda>0$ we have $\left|Z_{\alpha_{0}}^{h_{0}}(\lambda)\right| \leq A \lambda$. This technical condition is interesting because of the following theorem [BJR], which will be used in the proof of Theorem 7.2. The Stoltz condition is related to the "cone condition" of Nagel and Stein [NS] and of Sueiro [Su].

Theorem 7.1. Let $(X, \beta, \mu, T)$ denote a dynamical system, with set $X, a$-algebra of its subsets $\beta$, a measure $\mu$ defined on the measurable space $(X, \beta)$ such that $\mu(X)=1$ and a measurable, measure preserving map $T: X \rightarrow X$. Suppose $g$ is in $L^{1}(X, \beta, \mu)$ and that the sequence of pairs on natural numbers $\left(n_{l}, k_{l}\right)_{l=1}^{\infty}$ is Stoltz. Then

$$
\widetilde{m_{g}}(x):=\lim _{l \rightarrow \infty} \frac{1}{k_{l}} \sum_{j=1}^{k_{l}} g\left(T^{n_{l}+j} x\right)
$$

exists almost everywhere with respect to $\mu$.

Theorem 7.2. Let $f$ be a meromorphic function on $\mathbb{H}_{c}$ satisfying conditions (1), (2) and (3) of Theorem 1.1. Then if $\left(n_{l}, k_{l}\right)_{l \geq 1}$ is Stoltz, for any $s \in \mathbb{H}_{c} \backslash \mathbb{L}_{\sigma_{0}}$, we have

$$
\lim _{l \rightarrow \infty} \frac{1}{k_{l}} \sum_{j=1}^{k_{l}} f\left(s+i T_{\alpha, \beta}^{n_{l}+j}(x)\right)=\frac{\alpha}{\pi} \int_{\mathbb{R}} \frac{f(s+i \tau)}{\alpha^{2}+(\tau-\beta)^{2}} d \tau
$$

for almost all $x$ in $\mathbb{R}$. 
Proof. Let

$$
\widetilde{m_{l, f}}(x):=\frac{1}{k_{l}} \sum_{j=1}^{k_{l}} f\left(s+i T_{\alpha, \beta}^{n_{l}+j}(x)\right) \quad(l=1,2, \ldots)
$$

and let

$$
\widetilde{m_{f}}(x):=\lim _{l \rightarrow \infty} \frac{1}{k_{l}} \sum_{j=1}^{k_{l}} f\left(s+i T_{\alpha, \beta}^{n_{l}+j}(x)\right)
$$

Notice that

$$
\widetilde{m_{l, f}}\left(s+i T_{\alpha, \beta}(x)\right)-\widetilde{m_{l, f}}(x)=\frac{1}{k_{l}}\left(f\left(s+i T_{\alpha, \beta}^{n_{l}+k_{l}+1}(x)\right)-f\left(s+i T_{\alpha, \beta}^{n_{l}+1}(x)\right)\right) .
$$

This means that $\widetilde{m_{f}}\left(s+i T_{\alpha, \beta}(x)\right)=\widetilde{m_{f}}(s+i(x)) \mu_{\alpha, \beta}$ almost everywhere. As an ergodic dynamical system constant along almost all orbits must be constant, we must have $\widetilde{m_{f}}(x)=$ $\int_{\mathbb{R}} f(s+i x) d \mu_{\alpha, \beta}(x)$. We have therefore shown that

$$
\lim _{l \rightarrow \infty} \frac{1}{k_{l}} \sum_{j=1}^{k_{l}} f\left(s+i T_{\alpha, \beta}^{n_{l}+j}(x)\right)=\frac{\alpha}{\pi} \int_{\mathbb{R}} \frac{f(s+i \tau)}{\alpha^{2}+(\tau-\beta)^{2}} d \tau,
$$

for almost all $x$ in $\mathbb{R}$, as required.

7.2. Applications and moving average ergodic Theorems. We will forgo the proof of the Theorems below as they follow from those of Theorems 2.3, 2.4, 2.6, 2.7, 2.9 and 5.1 respectively via minor modification, in a similar way, using Theorem 7.1 and Theorem 7.2.

Theorem 7.3. Suppose $\left(n_{q}, k_{q}\right)_{q \geq 1}$ is Stoltz. Suppose $k$ is any non-negative integer. Then the statement, for any natural number $l$,

$$
\lim _{q \rightarrow \infty} \frac{1}{k_{q}} \sum_{j=1}^{k_{q}}\left|\zeta^{(k)}\left(s+i T_{\alpha, \beta}^{n_{q}+j}(x)\right)\right|^{l}=\frac{\alpha}{\pi} \int_{\mathbb{R}} \frac{\left|\zeta^{(k)}(s+i \tau)\right|^{l}}{\alpha^{2}+(\tau-\beta)^{2}} d \tau
$$

for $\mu_{\alpha, \beta}$-almost all $x$ in $\mathbb{R}$, is equivalent to the Lindelöf Hypothesis.

Theorem 7.4. Suppose $\left(n_{q}, k_{q}\right)_{q \geq 1}$ is Stoltz. Then for almost all $x$ in $\mathbb{R}$ with respect to Lebesgue measure we have

$$
\lim _{q \rightarrow \infty} \frac{1}{k_{q}} \sum_{j=1}^{k_{q}} \log \left|\zeta\left(\frac{1}{2}+\frac{1}{2} i T^{n_{q}+j} x\right)\right|=\sum_{\rho: \Re(\rho)>\frac{1}{2}} \log \left|\frac{\rho}{1-\rho}\right| .
$$

Again, if either side is zero, this is equivalent to the Riemann Hypothesis.

We now consider Dirichlet $L$-functions.

Theorem 7.5. Let $L(s, \chi)$ denote the L-series associated to the character $\chi$. Suppose $\left(n_{q}, k_{q}\right)_{q \geq 1}$ is Stoltz, and let $k$ be a nonnegative integer. Then,

(i) if $\chi$ is non-principal, for $s \in \mathbb{H}_{-\frac{1}{2}} \backslash \mathbb{L}_{1}$ we have

$$
\lim _{q \rightarrow \infty} \frac{1}{k_{q}} \sum_{q=1}^{k_{q}} L^{(k)}\left(s+i T_{\alpha, \beta}^{n_{q}+j}(x), \chi\right)=\frac{\alpha}{\pi} \int_{\mathbb{R}} \frac{L^{(k)}(s+i \tau, \chi)}{\alpha^{2}+(\tau-\beta)^{2}} d \tau
$$




$$
=L^{(k)}(s+\alpha+i \beta, \chi) \quad \text { for almost all } x \text { in } \mathbb{R},
$$

(ii) if $\chi$ is principal, for $s \in \mathbb{H}_{-\frac{1}{2}} \backslash \mathbb{L}_{1}$ we have

$$
\lim _{q \rightarrow \infty} \frac{1}{k_{q}} \sum_{j=1}^{k_{q}} L^{(k)}\left(s+i T_{\alpha, \beta}^{n_{q}+j}(x), \chi\right)=\frac{\alpha}{\pi} \int_{\mathbb{R}} \frac{L^{(k)}(s+i \tau, \chi)}{\alpha^{2}+(\tau-\beta)^{2}} d \tau,
$$

for almost all $x$ in $\mathbb{R}$.

We now consider the Hurwitz zeta function.

Theorem 7.6. Suppose $\left(n_{q}, k_{q}\right)_{q \geq 1}$ is Stoltz. For any s such that $\mathfrak{R}(s)>-\frac{1}{2}, s \neq 1,0 \leq a<1$ and $k$ a non-negative integer, we have

$$
\lim _{q \rightarrow \infty} \frac{1}{k_{q}} \sum_{j=1}^{k_{q}} \zeta^{(k)}\left(s+i T_{\alpha, \beta}^{n_{q}+j}(x), a\right)=\frac{\alpha}{\pi} \int_{\mathbb{R}} \frac{\zeta^{(k)}(s+i \tau, a)}{\alpha^{2}+(\tau-\beta)^{2}} d \tau,
$$

for almost all $x$ in $\mathbb{R}$.

In (7.9) the right hand side is given by (2.16), (2.17) and (2.18).

The analogue of Theorem 5.1 is now the following.

Theorem 7.7. Consider two ergodic dynamical systems $\left(X_{1}, \beta_{1}, \mu_{1}, T_{1}\right)$ and $\left(X_{2}, \beta_{2}, \mu_{2}, T_{2}\right)$, both on separable measure spaces. Suppose that $\mu_{1}$ and $\mu_{2}$ are non-atomic. Then if for a Stoltz sequence of integers $\left(n_{q}, k_{q}\right)_{q \geq 1}$ and each $f_{1} \in L^{p}\left(X_{1}, \beta_{1}, \mu_{1}\right)$ we have

$$
\lim _{q \rightarrow \infty} \frac{1}{k_{q}} \sum_{j=1}^{k_{q}} f_{1}\left(T_{1}^{n_{q}+j} x_{1}\right)=\int_{X_{1}} f_{1}\left(x_{1}\right) d \mu_{1},
$$

$\mu_{1}$ almost everywhere, then the same is true with 1 replaced by 2.

\section{SublineARITy AND THE RIEMANN ZETA FUNCTION}

In the same vein as Theorem 1.2, Theorem 1.3 and Theorem 1.4, now with peculiar sublinear sequences, the following Theorem can be deduced.

The following Lemma is taken from [LW2]. Let $I_{A}$ denote the indicator function of the set $A$.

Lemma 8.1. Suppose $k_{q}=k(q), q=1,2, \ldots$, with $k$ sub-linear such that $\sup _{u} k^{\prime}(u)$ is absolutely bounded. Also assume

$$
K(\theta)=\frac{1}{|\theta|} \int_{\min \left(\frac{1}{|\theta|}, 1\right)} \frac{k^{\prime}(u)}{|u|^{2}} d u+k\left(\frac{1}{|\theta|}\right)|\theta| I_{\{|\theta| \leq 1\}},
$$

is uniformly bounded on $\mathbb{R}$. Suppose $(X, \beta, \mu, T)$ is a dynamical system and $f \in L^{2}(X, \beta, \mu)$ and set

$$
g_{n}^{k}(f(x))=\frac{1}{n} \sum_{j=k_{n}}^{k_{n}+n-1} f\left(T^{j}(x)\right) .
$$

Then, for any strictly increasing sequence of natural numbers $\left(n_{q}\right)_{q \geq 1}$, there exists $C>0$ such that

$$
\left\|\left(\sum_{q \geq 1}\left|g_{n_{q+1}}^{k}(f)-g_{n_{q}}^{k}(f)\right|^{2}\right)^{\frac{1}{2}}\right\|_{2} \leq C\|f\|_{2}
$$


We now specialize to the case, $f(x)=\zeta(\sigma+i x)$ with $\sigma \in\left[\frac{1}{2}, 1\right)$.

Theorem 8.2. Suppose $k$ and $K$ are as in Lemma 8.1. Now set, with $\sigma \in\left[\frac{1}{2}, 1\right)$,

$$
G_{n, \sigma}^{k}(x)=G_{n}^{k}(\zeta(\sigma+i x))=\frac{1}{n} \sum_{j=k_{n}}^{k_{n}+n-1} \zeta\left(\sigma+i T^{j}(x)\right) .
$$

Then, for any strictly increasing sequence of natural numbers $\left(n_{q}\right)_{q \geq 1}$, there exists a constant $C>0$ such that

$$
\left\|\left(\sum_{q \geq 1}\left|G_{n_{q+1}, \sigma}^{k}(\zeta)-G_{n_{q}, \sigma}^{k}(\zeta)\right|^{2}\right)^{\frac{1}{2}}\right\|_{2} \leq \frac{C}{\pi \sigma}\left|\frac{\zeta(2 \sigma)}{2}+\frac{\zeta(2 \sigma-1)}{2 \sigma-1}\right| .
$$

\section{ACKNOWLEDGEMENTS}

We thank the referee for very detailed comments, substantially improving the presentation of this paper, and for bringing to our attention important additional references of material significance to this topic.

\section{REFERENCES}

[AdW] R.L. Adler and B. Weiss, The ergodic infinite measure preserving transformation of Boole, Israel J. Math. 16 (1973), 263-278.

[As] I. Assani, Convergence of the p-series for stationary sequences, New York J. Math. 3A (1997/98), Proceedings of the New York Journal of Mathematics Conference, June 9-13 (1997), 15-30.

[BSY] M. Balazard, E. Saias and M. Yor, Notes on the Riemann ל-function. II, Adv. Math. 143 (1999), no. 2, 284-287.

[BJR] A. Bellow, R. Jones and J. Rosenblatt, Convergence for moving averages, Ergod. Theory Dynam. Sys. 10 (1990), no. 1, 43-62.

[BL] A. Bellow and V. Losert, On sequences of zero density in ergodic theory, Conference in Modern Analysis and Probability (New Haven, Conn. 1982), 49-60, Contemp. Math. 26, Amer. Math. Soc., Providence RI, 1984.

[Be] B.C. Berndt, On the Hurwitz zeta-function, Rocky Mountain J. Math. 2 (1972), 151-157.

[Bo] G. Boole, On the comparison of transcendents and certain applications to the theory of indefinite integrals, Phil. Trans. Roy. Soc. London, 147, Part 11 (1857), 745-803.

[BKQW] M. Boshernitzan, G. Kolesnik, A. Quas and M. Wierdl, Ergodic averaging sequences, J. Anal. Math. 95 (2005), 63-103.

[Bou ] J. Bourgain, On the maximal ergodic theorem for certain subsets of the integers, Israel J. Math. Vol. 61, (1988), no. 1, 39-72.

[Br] W.E. Briggs, Some constants associated with the Riemann zeta-function, Michigan Math. J. 3 (1955/1956) 117-121.

[C] M. W. Coffey, Evaluation of some second moment and other integrals for the Riemann, Hurwitz, and Lerch zeta functions, (2005), ArXiv : 1101.5722v1.

[Coh] H. Cohen, Number theory. Vol. II. Analytic and modern tools, Graduate Texts in Mathematics 240 , Springer, New York, 2007.

[EW] M. Einsiedler and T. Ward, Ergodic Theory with a View Towards Number Theory, Springer, New York, 2011.

[G1] J.W.L. Glashier, On a theorem in definite integration, Quart. J. Pure and Appl. Math. 10 (1870), 347356.

[G2] J.W.L. Glashier, Note on certain theorems in definite integration, Messenger Math. 10 (1879), 63-74.

[HIKW] Y. Hashimoto, Y. Iijima, N. Kurokawa and M. Wakayama, Euler's constants for the Selberg and the Dedekind zeta functions, Bull. Belg. Math. Soc. Simon Stevin 11 (2004), 493-516. 
[IK] M. Ishibashi and S. Kanemitsu, Dirichlet series with periodic coefficients, Results Math. 35 (1999), $70-88$.

[JOP] B. Jamison, S. Orey and W. Pruitt, Convergence of weighted averages of independent random variables, Z. Wahrscheinlichkeitstheorie und Verw. Gebiete 4 (1965), 40-44.

[JKRW] R.L. Jones, R. Kaufman, J.M. Rosenblatt and M. Wierdl, Oscillation in ergodic theory, Ergodic Theory Dynam. Systems 18 (1998), no. 4, 889-935.

[JO ] R. L. Jones and J. Olsen, Subsequence ergodic theorem for operators, Israel J. Math. 77 (1992), no. $1-2,33-54$.

[Kt] Y. Katznelson, An introduction to Harmonic Analysis, Second corrected edition, Dover Publications, Inc., New York, 1976.

[KN] L. Kuipers and H. Niederreiter, Uniform Distribution of Sequences, John Wiley \& Sons, New York, 1974.

[LS] J. Lee and A.I. Suriajaya, An ergodic value distribution of certain meromorphic functions, J. Math. Anal. Appl. 445 (2017), no. 1, 125-138.

[LW1] M. Lifshits and M. Weber, Spectral regularization inequalities, Math. Scand. 86 (2000), no. 1, 75-99.

[LW2] M. Lifshits and M. Weber, Régularisation spectrale et propriétés métriques des moyennes mobiles, (French. English summary) [Spectral regularization and metric properties of moving averages], J. Anal. Math. 89 (2003), 1-14.

[LW3] M. Lifshits and M. Weber, Sampling the Lindelöf Hypothesis with the Cauchy random walk, Proc. Lond. Math. Soc. 98 (2009), 241-270.

[MSr] N. Maugmai and T. Srichan, The mean-value of meromorphic functions with respect to a generalized Boolean transformation, Acta Math. Sin. (Engl. Ser.) 35 (2019), no. 5, 662-670.

[MSt] B. Mazur and W. Stein, Prime Numbers and the Riemann Hypothesis, Cambridge University Press, 2016.

[NS] A. Nagel and E.M. Stein, On Certain Maximal Functions and Approach Regions, Adv. in Math. 54 (1984), 83-106.

[N] R. Nair, On uniformly distributed sequences of integers and Poincaré recurrence II, Indag. Math. (N. S.) 9 (1998), no. 3, 405-415.

[N2] R. Nair, On polynomial ergodic averages and square functions, Number theory and Polynomials, London Math. Soc. Lecture Note Ser., Cambridge Univ. Press, 352 (2008), 241-254.

[NW1] R. Nair and M. Weber, On variation functions for subsequence ergodic averages, Monatsh. Math. 128 (1999), no. 2, 131-150.

[NW2] R. Nair and M. Weber, On random perturbation of some intersective set, Indag. Math. (N.S) 15 (2004), no. 3, 373-381.

[RR] M.M. Rao and Z.D. Ren, Theory of Orlicz Spaces, Monographs and Textbooks in Pure and Applied Mathematics, Marcel Dekker, 1991.

[R] D. Redmond, A generalization of a theorem of Ayoub and Chowla, Proc. Amer. Math. Soc. 86 (1982) 574-580.

[Sa] S. Sawyer, Maximal inequalities of weak type, Ann. of Math. (2) 84 (1966), 157-174.

[Sr] T. Srichan, Sampling the Lindelöf Hypothesis for Dirichlet L-functions by the Cauchy random walk, Eur. J. Math. 1 (2015), 351-366.

[SW] E. Stein and G. Weiss, Introduction to Fourier Analysis on Euclidean Spaces, Princeton University Press, 1971.

[St1] J. Steuding, On the value-distribution of L-functions, Fiz. Mat. Fak. Moksl. Semin. Darb. 6 (2003) $87-119$.

[St2] J. Steuding, Sampling the Lindelöf Hypothesis with an ergodic transformation, RIMS Kôkyûroku Bessatsu B34 (2012), 361-381.

[Su] J. Sueiro, A note on maximal operators of Hardy-Littlewood type, Math. Proc. Cambridge Philos. Soc. 102 (1987), 131-134.

[T] E.C. Titchmarsh, The Theory of the Riemann Zeta-Function, second ed. (revised by D.R. Heath-Brown), Oxford Univ. Press, 1986.

[W] M. Weber, Dynamical systems and processes. IRMA Lectures in Mathematics and Theoretical Physics, 14. European Mathematical Society (EMS), Zürich (2009). 
$\ddagger$ Mathematical Sciences, The University of Liverpool, 1 Peach Street, Liverpool, L69 7ZL, U.K.

Email address: nair@liv.ac.uk

$\diamond$ LAMA, CNRS UMR 5127, Univ. Grenoble AlPes, Univ. Savoie Mont Blanc, F - 73000 ChAmbÉRY, FRANCE

Email address: Jean-Louis.Verger-Gaugry@univ-smb.fr

† IRMA, 10 Rue du Général-Zimmer, 67084 Strasbourg, Cedex, France.

Email address: michel. weber@math. unistra.fr 